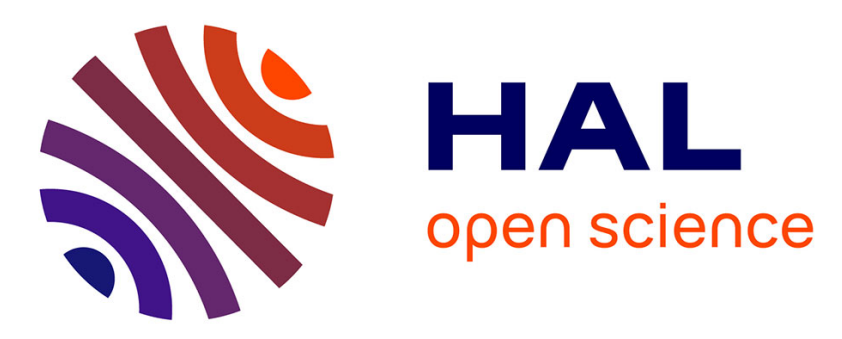

\title{
Martian ice cloud distribution obtained from SPICAM nadir UV measurements
}

Nina Mateshvili, Didier Fussen, Filip Vanhellemont, Christine Bingen, Jan

Dodion, Franck Montmessin, Severine Perrier, Emmanuel Dimarellis, Jean-Loup Bertaux

\section{To cite this version:}

Nina Mateshvili, Didier Fussen, Filip Vanhellemont, Christine Bingen, Jan Dodion, et al.. Martian ice cloud distribution obtained from SPICAM nadir UV measurements. Journal of Geophysical Research. Planets, 2007, 112 (E7), pp.E07004. 10.1029/2006JE002827 . hal-00164681

\section{HAL Id: hal-00164681 \\ https://hal.science/hal-00164681}

Submitted on 9 Oct 2020

HAL is a multi-disciplinary open access archive for the deposit and dissemination of scientific research documents, whether they are published or not. The documents may come from teaching and research institutions in France or abroad, or from public or private research centers.
L'archive ouverte pluridisciplinaire HAL, est destinée au dépôt et à la diffusion de documents scientifiques de niveau recherche, publiés ou non, émanant des établissements d'enseignement et de recherche français ou étrangers, des laboratoires publics ou privés. 


\title{
Martian ice cloud distribution obtained from SPICAM nadir UV measurements
}

\author{
Nina Mateshvili, ${ }^{1}$ Didier Fussen, ${ }^{1}$ Filip Vanhellemont, ${ }^{1}$ Christine Bingen, ${ }^{1}$ Jan Dodion, ${ }^{1}$ \\ Franck Montmessin, ${ }^{2}$ Severine Perrier, ${ }^{2}$ Emmanuel Dimarellis, ${ }^{2}$ and Jean-Loup Bertaux ${ }^{2}$ \\ Received 14 September 2006; accepted 26 March 2007; published 21 July 2007.
}

[1] The Spectroscopy for the Investigation of the Characteristics of the Atmosphere of Mars (SPICAM) instrument on board Mars Express has successfully performed one Martian year of measurements. Nadir UV (200-310 nm) measurements allowed it to build maps of ice cloud optical depth distribution for all seasons. The development and decay of the aphelion cloud belt (ACB) and polar hoods were observed. The characteristic values of the cloud optical thickness were $0.1-0.3$ at the early stage of the ACB formation in the solar longitude range $\mathrm{L}_{\mathrm{s}}=20-60^{\circ}$. After $\mathrm{L}_{\mathrm{s}}=93^{\circ}$, the well-developed ACB showed cloud optical thicknesses varying between 0.3 and 0.8 . The ACB quickly decayed after $\mathrm{L}_{\mathrm{s}}=140^{\circ}$. Both polar hoods were observed during their development and decay stages, showing cloud optical thicknesses of about 0.35 . The north polar hood started to develop at $\mathrm{L}_{\mathrm{s}}=160^{\circ}$ and the south one at $\mathrm{L}_{\mathrm{s}}=330^{\circ}$. Estimates of water content in the ice clouds gave values of $0.35-1.8 \mathrm{gm}^{-2}$ for ACB and $0.4 \mathrm{gm}^{-2}$ for the polar hoods. A comparison with water vapor abundance showed that only a small fraction $(10-20 \%$ for $\mathrm{ACB}$ and $30 \%$ for the polar hoods) of total water content in the atmosphere was accumulated in clouds. The Martian surface albedo at the wavelength $300 \mathrm{~nm}$ appeared very low (0.004-0.018) and exhibited anticorrelation with the visual albedo consistent with optical properties of iron oxides abundant in Martian soils. The investigation of a regional dust storm allowed the estimation of dust optical parameters at the wavelength $300 \mathrm{~nm}$ (asymmetry factor $\mathrm{g}_{\mathrm{d}}=0.8$ and single scattering albedo $\sigma_{\mathrm{d}}=0.6$ ).

Citation: Mateshvili, N., D. Fussen, F. Vanhellemont, C. Bingen, J. Dodion, F. Montmessin, S. Perrier, E. Dimarellis, and J.-L. Bertaux (2007), Martian ice cloud distribution obtained from SPICAM nadir UV measurements, J. Geophys. Res., 112, E07004, doi:10.1029/2006JE002827.

\section{Introduction}

[2] The ice clouds play an important role in Martian climate. They are closely related to the Martian atmospheric circulation and the water vapor cycle. The process of cloud formation also plays an important role in the removal of atmospheric dust particles, which serve as condensation nuclei and subsequently precipitate together with water ice.

[3] Three main types of cloudiness are usually distinguished on Mars: the north and south polar hoods, the aphelion cloud belt (ACB) and also isolated orographic clouds above the major volcanoes. The Martian clouds were intensively investigated by both telescopic and spacecraft observations in different wavelength domains. Tamppari et al. [2000] used Viking Infrared Thermal Mapper (IRTM) data. Pearl et al. [2001] mapped Martian clouds using Thermal Emission Spectrometer (TES). M. D. Smith et al. [2003] used thermal infrared images taken by the Thermal Emission Imaging System (THEMIS) on board the Odyssey

\footnotetext{
${ }^{1}$ Belgian Institute for Space Aeronomy, Brussels, Belgium.

${ }^{2}$ Service d'Aeronomie du CNRS/IPSL, Verrières-le-Buisson, France.
}

Copyright 2007 by the American Geophysical Union. 0148-0227/07/2006JE002827 spacecraft for the quantitative retrieval of water ice optical thicknesses. These last data proved to be a good complement to TES measurements because they where sampled during later local time and reflect diurnal evolution of clouds demonstrating a significant rise of their optical depth in the late afternoon (THEMIS) in comparison with the early afternoon (TES). The most comprehensive cloud maps were created by Wang and Ingersoll [2002] using Mars Orbiter Camera (MOC) red and blue low-resolution images of the Martian surface. Benson et al. [2003] used the same MOC observations for detailed investigation of the cloud seasonal behavior in the Tharsis and Valles Marineris regions. James et al. [1994] and Clancy et al. [1996] used Hubble Space Telescope (HST) observations at visible and ultraviolet wavelengths for cloud detection. Clancy et al. [1996] supposed that the ACB originates because of condensation of water vapor raised by the ascending branch of the Hadley circulation. This result was confirmed by Richardson and Wilson [2002] and Richardson et al. [2002] who presented a general circulation model study of cloud formation. He also demonstrated that the decay of the ACB in mid northern summer is related to an increase in air temperature, rather than to decreases in the water vapor supply or in the intensity of Hadley upwelling flux. 


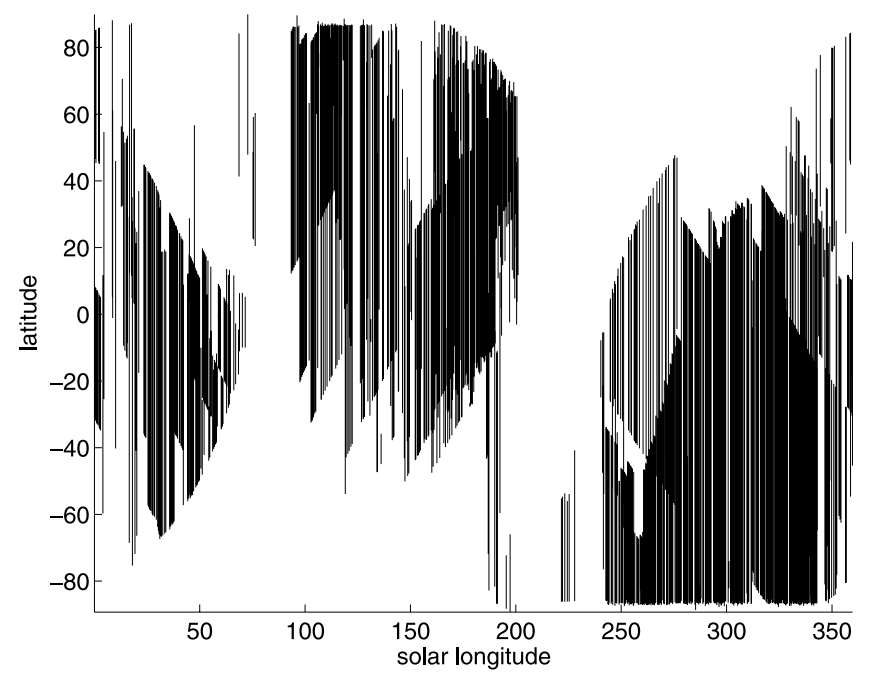

Figure 1. Orbit distribution as a function of the latitude and the solar longitude. Only parts of orbits with $S Z A<85^{\circ}$ are presented.

Montmessin et al. [2004] investigated the effect of the water ice clouds transport on the seasonal evolution of the water cycle.

[4] Wolff et al. [1999] used HST observations to investigate seasonal and interannual variations of the ACB. Clancy et al. [2003] and Wolff and Clancy [2003] used TES observations to estimate ice and dust particle size. They discovered the presence of two distinct populations of ice clouds: with particle effective radius $1-2 \mu \mathrm{m}$ and 3-4 $\mu \mathrm{m}$.

[5] Smith [2004] considered the interannual variability of water ice optical depth (as well as of dust, water vapor and surface temperature) from TES measurements.

[6] In this paper we present the Martian cloud distribution as it was observed in the Spectroscopy for the Investigation of the Characteristics of the Atmosphere of Mars (SPICAM) experiment. The SPICAM instrument is a two-channel (UV and IR) spectrometer on board Mars Express. Here we consider only nadir measurements in the SPICAM UV

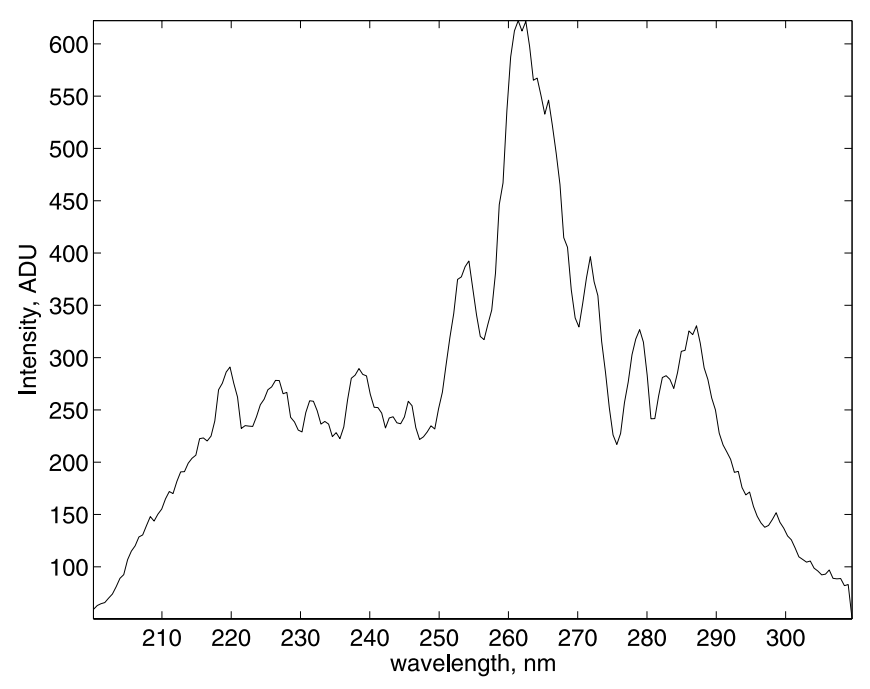

Figure 2. A typical raw SPICAM spectrum.
Table 1. Wavelength Subdomains Considered for SPICAM Spectra Analysis

\begin{tabular}{lc} 
& Wavelength Domain, nm \\
\hline Averaged signal $S_{\mathrm{av}}$ & $200-310$ \\
"Blue" band $\mathrm{S}_{\mathrm{b}}$ & $212-215$ \\
"Green" band $\mathrm{S}_{\mathrm{g}}$ & $250-260$ \\
"Red" band $\mathrm{S}_{\mathrm{r}}$ & $295-308$ \\
\hline
\end{tabular}

channel. A detailed description of the instrument is given by Bertaux et al. [2000]. The UV channel covers the 118$320 \mathrm{~nm}$ wavelength range but in this investigation we analyze only the range $200-320 \mathrm{~nm}$ because of the strong $\mathrm{CO}_{2}$ absorption band located at the shorter wavelengths. Twenty CCD lines were used to register twenty spectra in each single measurement. At readout of the data they were binned over four pixels in each CCD columns providing five averaged spectra. Each of them collected light reflected from slightly different areas of the Martian surface. The spectra had average resolution $1.2 \mathrm{~km}$ across the track and $2.4 \mathrm{~km}$ along the track [Perrier et al., 2006]. The measurements were made every second. In this paper we consider the period from 9 January 2004 to 27 January 2006 (orbits $8-2625$ ) that covers one Martian year (MY27, according to chronology proposed by Clancy et al. [2000]) starting from solar longitude $L_{s}=331^{\circ}$, MY 26 and finishing at $L_{s}=3^{\circ}$, MY 28.

\section{Observations and Cloud Detection}

\subsection{Temporal and Spatial Coverage}

[7] One of the important characteristics of the SPICAM data set is the spatial and temporal distribution of the orbits. Figure 1 shows the orbit distribution with respect to solar longitude. We consider only the measurements with solar zenith angles $(S Z A)$ less than $85^{\circ}$. For future reference, we give here the ranges of $L_{S}$ for the Martian seasons in the northern hemisphere: spring $\left(L_{s}=0-90^{\circ}\right)$, summer $\left(L_{s}=90-\right.$ $\left.180^{\circ}\right)$, fall $\left(L_{s}=180-270^{\circ}\right)$ and winter $\left(L_{s}=270-360^{\circ}\right)$. There are gaps in the measurements for northern latitudes higher than $50^{\circ}$ during the periods of late north fall and winter and for southern latitudes higher than $-50^{\circ}$ during the periods of late south fall and winter. These distributions should be kept in mind when considering the formation of north and south polar hoods. There are also gaps in the measurements between $L_{s}=73^{\circ}$ and $L_{s}=90^{\circ}$ and between $L_{S}=200^{\circ}$ and $L_{S}=240^{\circ}$.

\subsection{Qualitative Analysis of the Measurements}

[8] In this section, a qualitative analysis of the data set is performed for the uncalibrated data expressed in ADU (analog to digital units). Figure 2 shows a typical raw SPICAM spectrum. It makes sense to perform such an analysis before applying a retrieval procedure to achieve a better understanding of influencing factors and the way they affect the measured spectra. This would help us to design a better strategy of the subsequent retrieval of the ice cloud optical thickness.

[9] For further analysis, all spectra of each orbit were binned into a few subdomains of wavelengths (Table 1) and the data were averaged over each of them. The averaged values were considered as a function of latitude. 


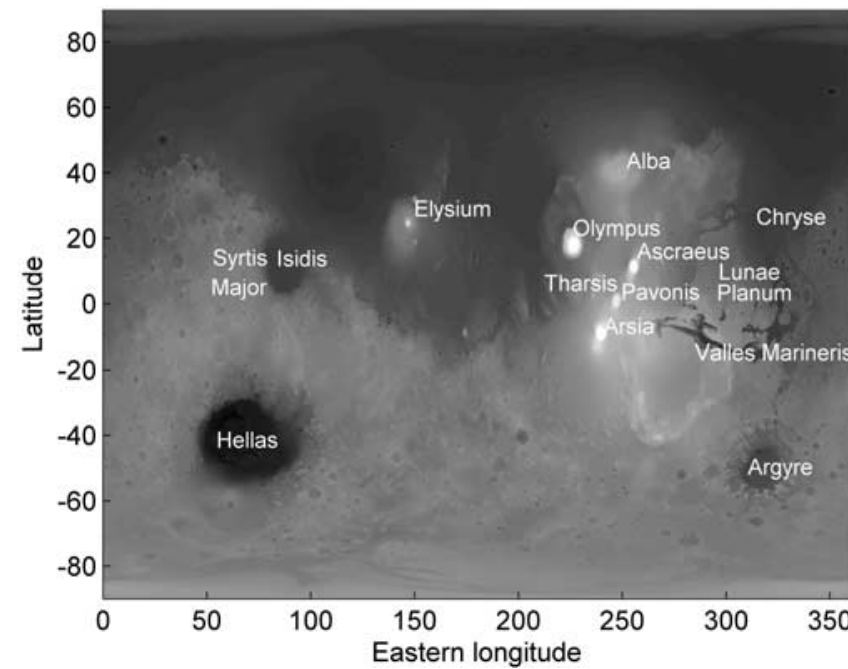

Figure 3. Mars Laser Altimeter map.

[10] The surface pressure is the main feature that influences the magnitude of the averaged signals $S_{a v}, S_{b}, S_{g}, S_{r}$ due to strong Rayleigh scattering in the UV. The sharp Martian relief is associated with strong differences of the surface pressure from $1 \mathrm{mbar}$ above the highest volcano Olympus Mons to 12 mbar in deep Hellas Planitia (Figure 3). The averaged signals quickly drop above mountains and rise above deep valleys. Figures $4 \mathrm{a}$ and $5 \mathrm{a}$ show two representative examples of this dependence. The $S_{r}$ has a minimum above Olympus Mons, and maximum above the deep canyon Valles Marineris (the corresponding altitudes are obtained from the Mars Orbiter Laser Altimeter (MOLA) altitudinal map [D. Smith et al., 2003]). The same behavior is observed in the averaged signals $S_{b}, S_{g}, S_{a v}$

[11] The change of the surface pressure causes changes not only in the magnitude of $S_{r}$ but also in the spectrum slope and it is useful to introduce the color ratio "red" to "blue" $R_{r b}=S_{r} / S_{b} . R_{r b}$ increases above the mountains (Figure $4 \mathrm{~b}$ ) and decreases above the valleys (Figure $5 \mathrm{~b}$ ). The alteration of $R_{r b}$ is caused by the concurrence between two processes with essentially different wavelength dependence: scattering on air molecules and surface reflection.
Above deep valleys, where the surface pressure is high, Rayleigh scattering, which is proportional to $\lambda^{-4}$, makes the main contribution to the observed brightness and causes a decrease of $R_{r b}$. On the contrary, above mountains $R_{r b}$ increases because the surface reflection contribution becomes more important because of decrease of the surface pressure. The surface albedo increases with $\lambda$ because of high iron oxides abundance in the Martian soil which makes the Martian surface red in visible wavelengths and dark in the UV [Bell, 1996].

[12] The Martian surface albedo exhibits significant variations. For example, the spikes on the left of Figure 4a show how bright ices manifest themselves as distinct maxima. The bare ground albedo also varies with geographical coordinates. Moreover, the Martian surface albedo is essentially non-Lambertian. Hapke [1986] described the opposition effect which consists in an increase of surface albedo at small phase angles (angles between the incident and the scattered light beams) for sandy or rough surfaces. The effect arises mainly because of the fact that interiors between sand particles become sunlit at small phase angles. Thorpe [1982] used the opposition effect for investigation of Martian soil properties.

[13] The obvious result of an increase of the surface albedo is an increase of $S_{a v}$ (as well as $S_{r}, S_{b}$ and $S_{b}$ ). Less trivial is the observed increase of $R_{r b}$. The reason is the same as the reason of the $R_{r b}$ increase above mountains: The contribution of the surface reflection to the observed brightness becomes more important in comparison with Rayleigh scattering.

[14] The considered range of wavelengths overlaps with the ozone Hartley band. On Mars, ozone is abundant mainly in polar regions during winter time. The total column amount for the considered period did not exceed the value about $33 \mu \mathrm{m}$-atm $(10 \mu \mathrm{m}$-atm $=1$ DU) [Perrier et al., 2006] and was much lower during most of the year. Figure 6 presents the modeled radiance factor (see section 4.1 for the definition of the radiance factor) for different values of ozone. It is clear that ozone affects only slightly the radiance factor at $300 \mathrm{~nm}$ (i.e., $S_{r}$ ) and a little bit more at $210 \mathrm{~nm}$ $\left(S_{b}\right)$. So $R_{r b}$ will undergo a slight increase due to the presence of ozone. The variations of ozone can be estimated using another green to red color ratio $R_{g r}$.
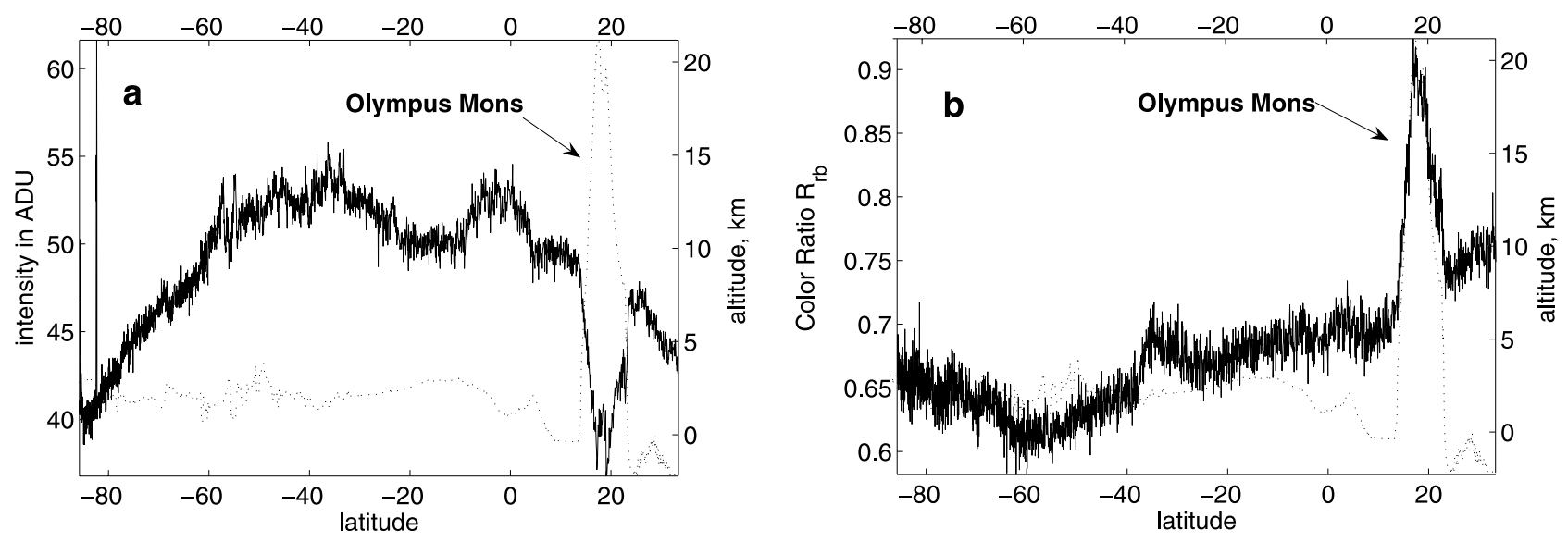

Figure 4. (a) "Red" SPICAM spectral band $S_{r}$ (solid line) and corresponding surface altitudes (dotted line) for orbit 2025 versus latitude. (b) Color ratio $R_{r b}$ for the same orbit. 

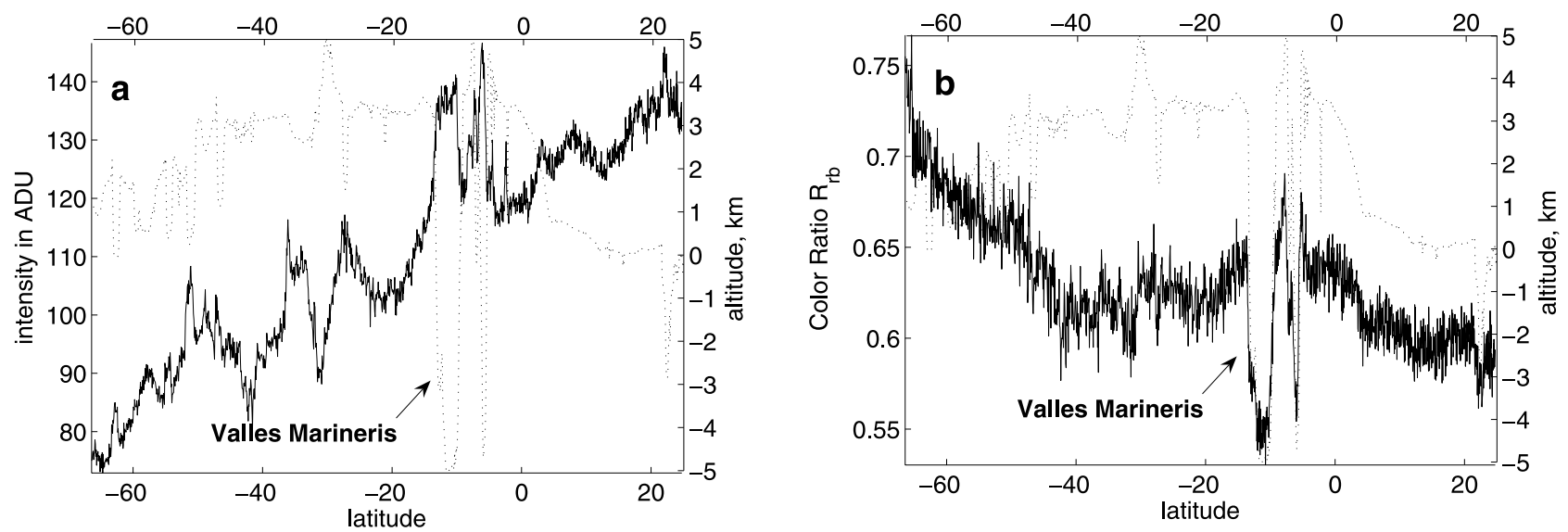

Figure 5. Same as Figure 4 for orbit 308.

[15] The Martian dust will be considered in section 3.2. The behavior of the averaged signals and color ratios are summarized in Table 2.

\section{Detection of Ice and Dust Clouds Using the Raw Data}

\subsection{Ice Cloud Detection}

[16] In this section a simple method of cloud detection from uncalibrated data is described. Because of the computing cost of a radiative transfer computation, some preliminary analysis may be useful. In most cases the Martian ice clouds are quite prominent in the UV because of their high albedo in comparison with the low albedo of the surface [Clancy et al., 1996; Petrova et al., 1996]. The main difficulty in cloud detection is due to the strong dependence of the UV reflectivity not only on the albedo but also on the surface pressure as it was discussed above. The following strategy was used to detect clouds. First, we consider the easiest case: detection of clouds above mountains. In this case we compare appropriate surface altitudes obtained from MOLA map and averaged signal $S_{a v}$ (or $S_{r}$ ). The increase of the signal above mountains instead of an expected decrease is interpreted as a cloud. Figure 7a shows a distinct maximum above Olympus Mons that was detected at orbit 1177. Comparison with Figure 4a (which shows a minimum) above the mountain confirms that we can associate the maximum with the presence of a cloud. We can see that it is almost as bright as the ices of the north pole (in the right of Figure $7 \mathrm{a}$ ). The presence of the cloud can be confirmed using MOC wide-angle images (M. C. Malin et al., Malin Space Science Systems Mars Orbiter Camera Image Gallery, available at http://www.msss.com/moc gallery/). Fortunately, the images of Mars by MOC are available in most cases at the SPICAM measurement time. The MOC images are obtained in two spectral channels: blue $(400-450 \mathrm{~nm})$ and red $(575-625 \mathrm{~nm})$. The MOC blue channel was used by Wang and Ingersoll [2002] for ice cloud detection. Figure 8 represents the blue and red channel MOC images acquired on the same day of the orbit 1177. The blue image shows a prominent cloud above Olympus Mons and confirms our results. We should mention that the $R_{r b}$ color ratio also increases in case of cloud presence as is shown in Figure $7 \mathrm{~b}$.
[17] More sophisticated is the detection of clouds above plains and valleys, because usually they are not as prominent as those above mountains and the interpretation is not so obvious. In such cases, it appeared helpful to consider also the increase of color ratio $R_{r b}$ as one more indication of cloud presence. The increase of $R_{r b}$ in presence of an ice cloud (Figure 9, dotted curve) is confirmed by the radiative transfer modeling (see section 4 for the forward model details).

[18] In the most complex cases it is necessary to compare different orbits which cross the same area. The clouds above the Hellas basin may serve as a typical example of such a complex case. Finally, we formulate the following procedure of cloud detection from the raw uncalibrated data.

[19] The first step is to detect an increase of $S_{a v}$. The second step is to compare the detected increase with the MOLA topography map to exclude the presence of a valley. The increase of color ratio $R_{r b}$ confirms that the feature is likely a cloud. The seasonal boundaries of polar caps should be checked because it is often difficult to distinguish between ice and bright ice clouds. It should be kept in mind that some Martian soils can appear unexpectedly

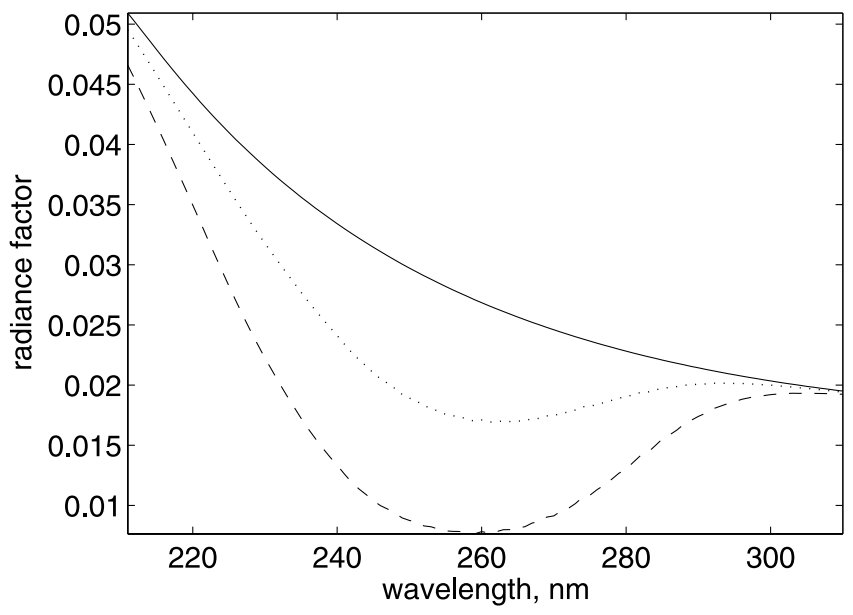

Figure 6. Modeled radiance factor without ozone (solid line), in presence of $10 \mu \mathrm{m}$-atm of ozone (dotted line) and in presence of $33 \mu \mathrm{m}$-atm of ozone (dashed line). 
Table 2. Influence of Increasing Different Factors on the Averaged Signals and Color Ratios of SPICAM Spectra

\begin{tabular}{|c|c|c|c|c|c|c|}
\hline Factor & $\begin{array}{l}\text { Averaged } \\
\text { Signal } \mathrm{S}_{\mathrm{av}} \\
\end{array}$ & $\begin{array}{l}\text { "Blue" } \\
\text { Band } \mathrm{S}_{\mathrm{b}}\end{array}$ & $\begin{array}{l}\text { "Green" } \\
\text { Band } \mathrm{S}_{\mathrm{g}}\end{array}$ & $\begin{array}{l}\text { "Red" } \\
\text { Band } \mathrm{S}_{\mathrm{r}}\end{array}$ & $\begin{array}{l}\text { Color Ratio } \\
\mathrm{R}_{\mathrm{rb}}=\mathrm{S}_{\mathrm{r}} / \mathrm{S}_{\mathrm{b}}\end{array}$ & $\begin{array}{l}\text { Color Ratio } \\
\mathrm{R}_{\mathrm{gr}}=\mathrm{S}_{\mathrm{g}} / \mathrm{S}_{\mathrm{r}}\end{array}$ \\
\hline Surface pressure & increase & increase & increase & increase & decrease & increase \\
\hline Albedo & increase & increase & increase & increase & increase & decrease \\
\hline Cloud & increase & increase & increase & increase & increase & decrease \\
\hline Ice & increase & increase & increase & increase & increase & decrease \\
\hline Dust & decrease & strong decrease & decrease & constant & increase & decrease \\
\hline Ozone & decrease & slight decrease & strong decrease & constant & slight increase & strong decrease \\
\hline
\end{tabular}

bright in the UV, and in all suspicious cases it is necessary to intercompare different orbits. It is also useful to check the value of phase angle to avoid the opposition effect.

[20] Some examples of bright orographic clouds are presented in Figure 10. A bright cloud above Elysium Mons (Figure 10a) was detected at orbit $980, L_{s}=105^{\circ}$, in the period when the aphelion cloud belt was already well developed. Figure 10b shows a cloud above Alba Patera and cloud part above Tharsis region (orbit 902, $L_{s}=95^{\circ}$ ). Tiny clouds above Arsia Mons were detected at $L_{s}=310^{\circ}$ (orbit 2274, Figure 10c). These clouds are quite distinguishable and easy to detect.

[21] Another group of clouds are clouds which belong to the aphelion cloud belt. Most of them are very prominent as we can see in the Tharsis region (Figures $10 \mathrm{~b}$ and $10 \mathrm{~d}$ ).

[22] In the polar regions, one has to distinguish between north polar summer clouds and clouds which form the north and south polar hoods. The last ones are less prominent than $\mathrm{ACB}$ clouds and their detection from the raw data is often complicated by large solar zenith angles.

[23] We should stress that the qualitative procedure for cloud detection was used only on the first stage of analysis when the retrieval scheme was developed. The final cloud data set was obtained automatically, using the radiative transfer code.

\subsection{Dust Cloud Detection}

[24] Although this paper is mainly focused on ice clouds, we should mention some dust features. The understanding of the dust role in scattering and absorption in the UV is important for the correct retrieval of ice cloud optical depth. In visual wavelength ranges, both dust and ice clouds cause an increase of brightness. Cantor et al. [2001] used MOC red and blue channel images to detect dust clouds. The dust clouds were detected as bright features in red MOC channel and less bright in blue one.

[25] In this section we analyze how a dust storm manifested itself in the UV. During the opposition of Mars in October 2005 the planet was actively monitored by the Hubble telescope and by ground-based instruments on the Earth. A regional dust storm was detected during the observational campaign. The storm originated in Chryse and later moved southward [McKim, 2006]. SPICAM crossed one of the branches of the storm on 24 October (orbit 2284, $L_{s}=312^{\circ}$ ) in the east of the Argyre basin (Figure 11). The presence of a dust cloud in this place at the same day is confirmed by a telescopic image obtained by $\mathrm{J}$. Warren (inset in Figure 11, http://marswatch.amaonline. com/mars_images_2003a.htm). The orbit 2262 (18 October, $\left.L_{s}=308^{\circ}\right)$ crossed almost the same area as the orbit 2284 only 6 days before the dust storm. Figure 12 shows two ratios: the ratio between the "red" bands of SPICAM spectra for orbits 2264 and 2284 (solid line) and that between "blue" bands for the same orbits (dotted line). Although the "red" ratio shows practically no change, the "blue" ratio clearly indicates the presence of dust as a sharp decrease of the signal. This means two things. First, that the "red" SPICAM band is minimally sensitive to the presence of dust. Second, that dust shows strong extinction in the "blue" SPICAM band. Thus, in a qualitative way it is
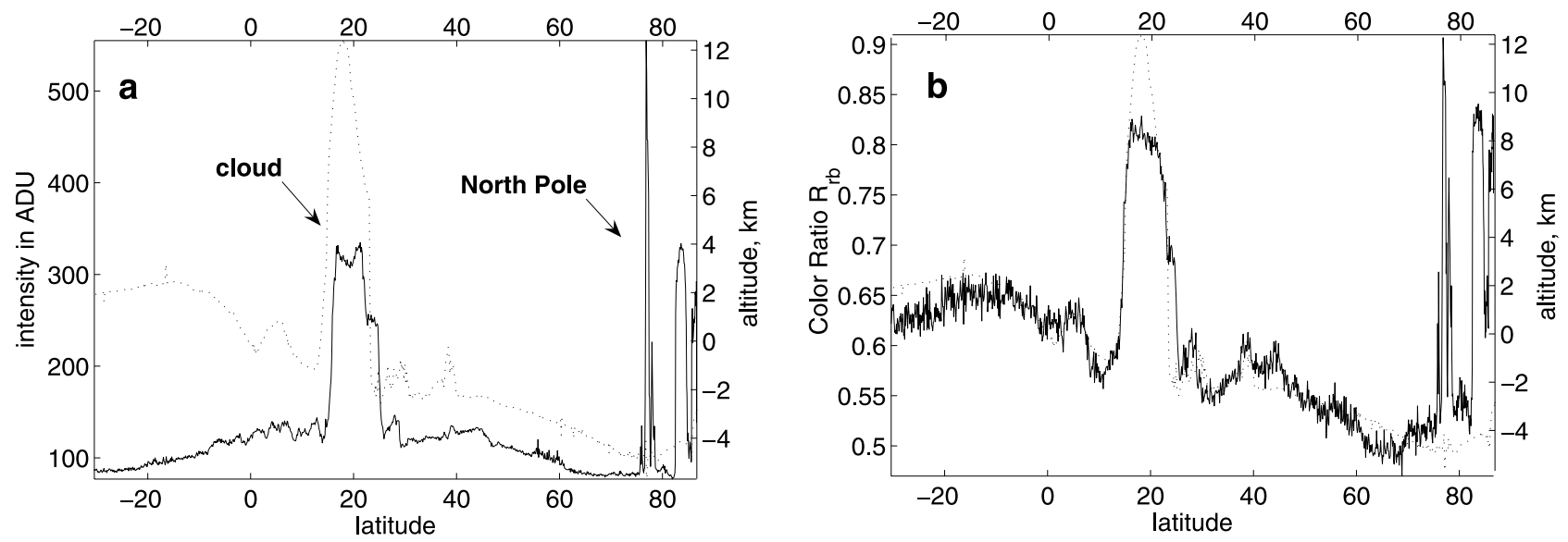

Figure 7. Ice cloud above Olympus Mons (orbit 1177). (a) "Red" SPICAM band $S_{r}$ versus latitude (solid line) and corresponding surface altitude (dotted line). (b) Color ratio $R_{r b}$ versus latitude (solid line) and surface altitude (dotted line). 


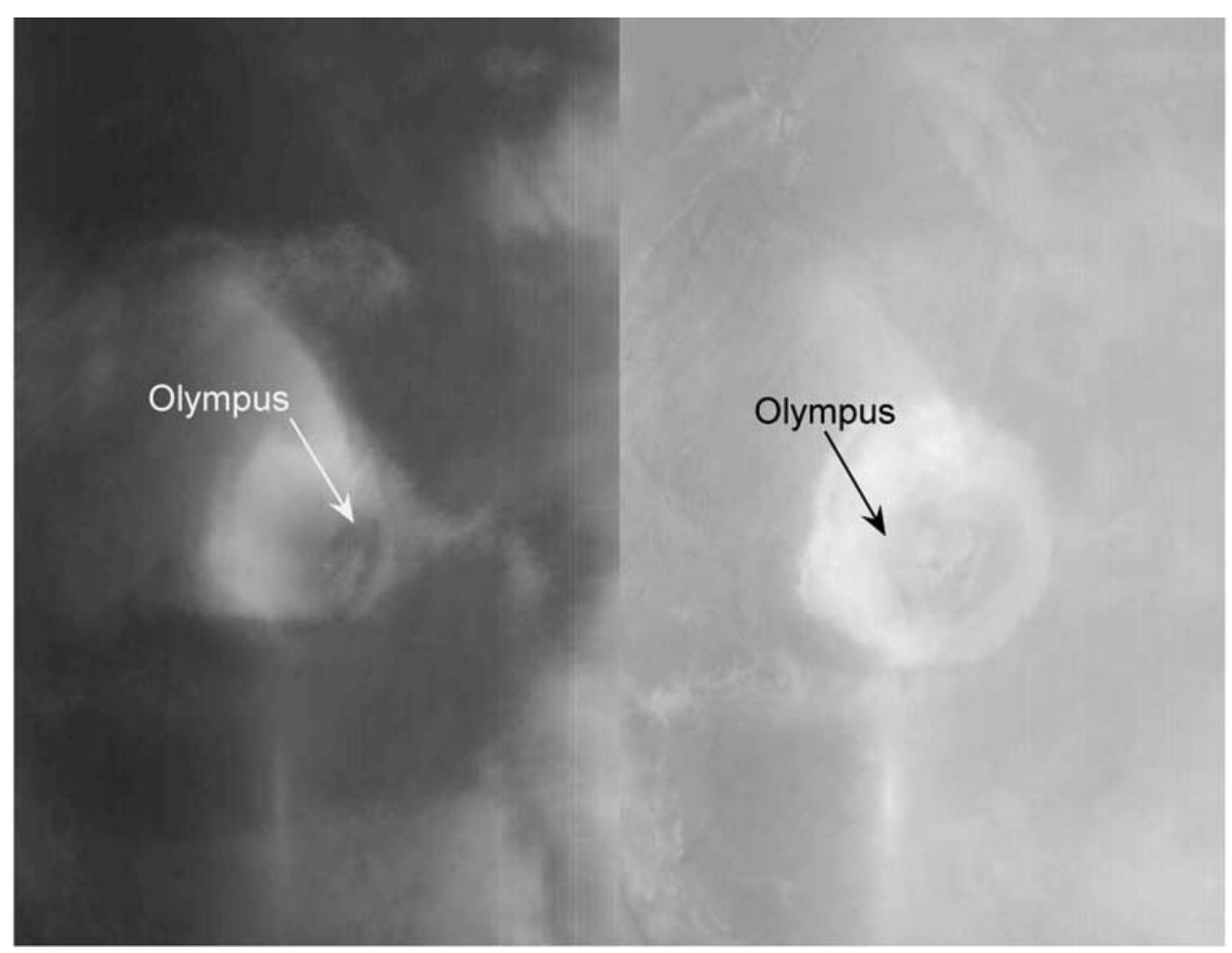

Figure 8. (left) Blue (S0100764) and (right) red (S0100763) MOC images of Olympus Mons which were acquired in the same day as orbit 1177 (see Figure 7).

possible to elaborate simple criteria for dust cloud detection from the raw data, i.e., a decrease in the "blue" band $S_{b}$ and a significant increase of color ratio $R_{r b}$ (because the "red" band is practically not affected by dust). The increase of $R_{r b}$ in presence of dust cloud (Figure 9, crosses) is confirmed by the radiative transfer modeling (see section 4 for the forward model details).

[26] In section 4.4 the dust optical properties will be analyzed in more detail and some quantitative characteristics of the dust optical parameters will be estimated.

\section{Cloud Optical Thickness Retrieval}

\subsection{Calibration}

[27] The first step in the data processing is to convert raw data expressed in ADU to the radiance factor $I / F$. $\pi F$ is the solar flux at the top of the atmosphere at the particular wavelength and $I$ is light intensity scattered in the direction of observation. The data were corrected for dark current and nonuniformal pixel sensitivity. The special shape of slit, which is a combination of a wide slit dedicated to limb measurements and of a narrow slit for nadir measurements, appeared to be a source of stray light that penetrates through the wide slit during nadir measurements. A special procedure for stray light correction is described by Bertaux et al. [2006]. The spectral sensitivity of the UV channel was obtained from SPICAM UV stellar observations outside the atmosphere. More details of the data processing are described by Perrier et al. [2006].

\subsection{Radiative Transfer Model}

[28] For the retrieval of cloud optical thickness, a multiscattering radiative transfer code for plane-parallel atmos- pheres was used. The code is based on Spherical Harmonic Discrete Ordinate Method SHDOM [Evans, 1998]. The model of the Martian atmosphere which is used as an input to the radiative transfer code is described by Perrier et al. [2006]. Here we only briefly mention some important details. The ground albedo was supposed Lambertian. The non-Lambertian properties of the ground albedo were taken into account separately (see below). The surface pressure was taken from the GCM calculations [Forget et al., 1999], and the pressure profile was calculated using the hydrostatic equation. The surface temperature and the temperature

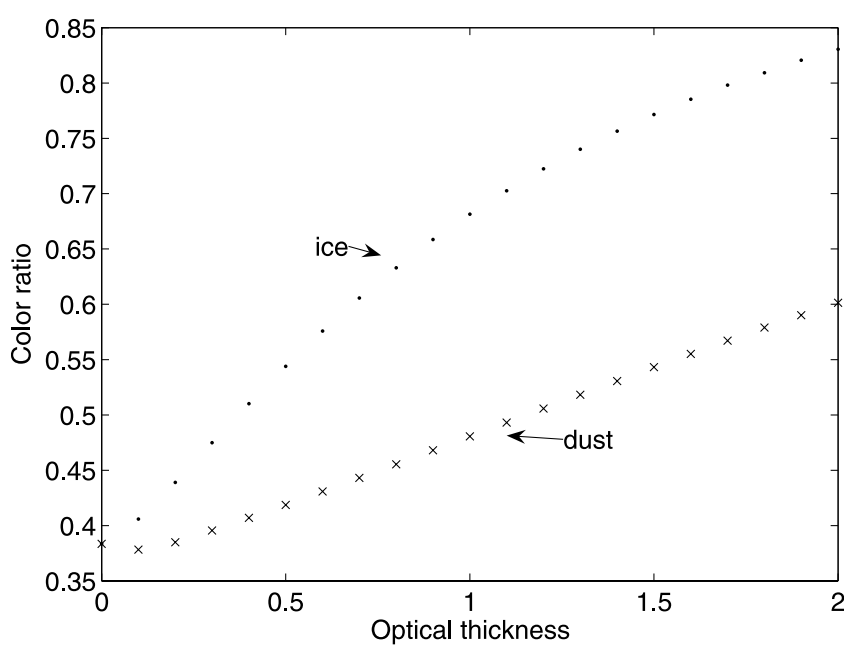

Figure 9. Color ratio $R_{r b}$ in presence of ice or dust cloud versus cloud optical thickness. 

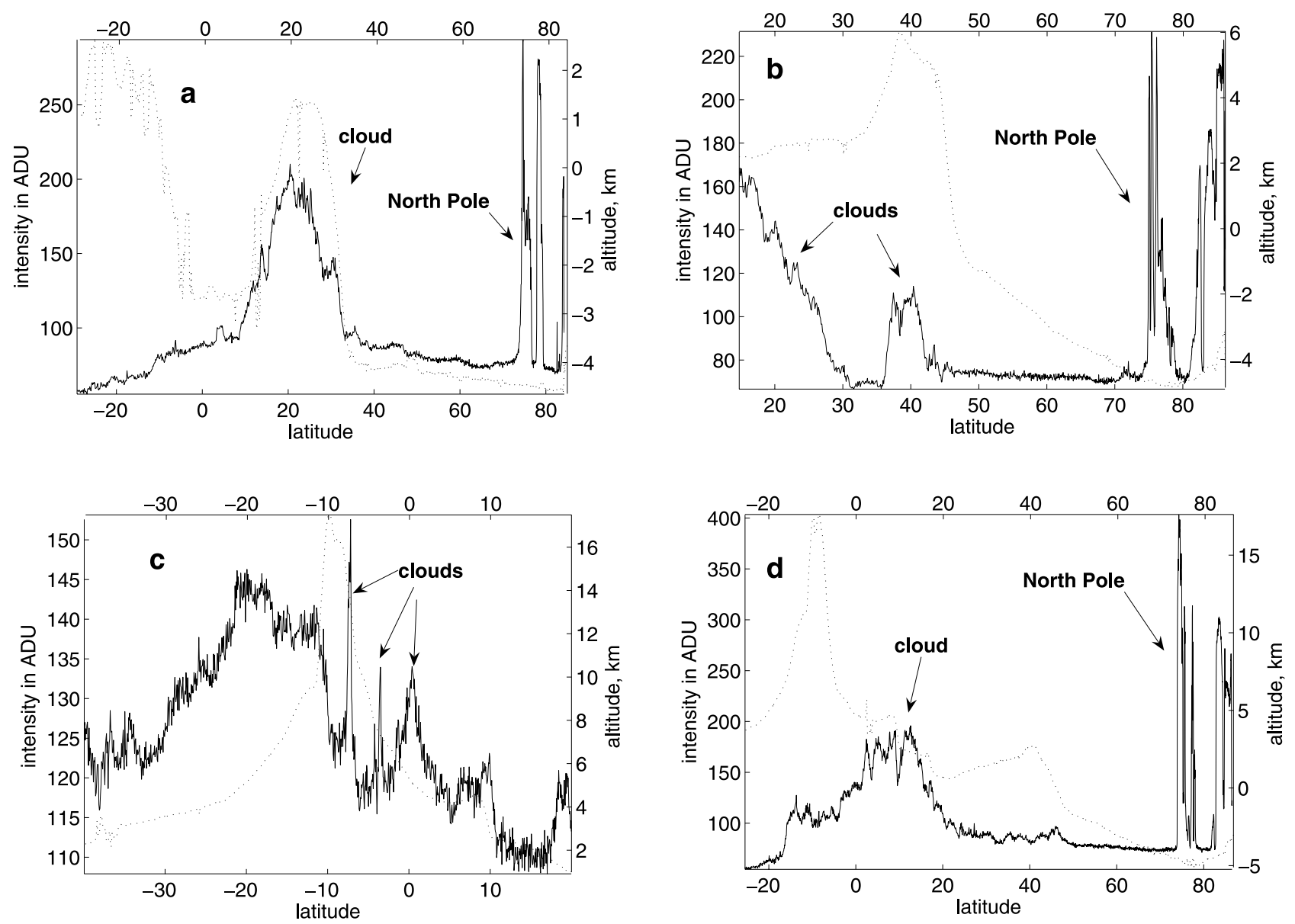

Figure 10. A few examples of ice clouds. "Red" SPICAM band versus latitude is indicated by the solid line, and appropriate surface altitude is indicated by the dotted line. (a) Ice cloud above Elysium Mons (orbit 980, $L_{s}=105^{\circ}$ ), peaks in the right are ices of the north pole; (b) cloud above Alba Patera and a part of the aphelion cloud belt (orbit 902, $L_{s}=95^{\circ}$ ); (c) tiny clouds above Arsia Mons (orbit 2274, $L_{S}=310^{\circ}$ ); and (d) a part of aphelion cloud belt in Tharsis region (orbit 1001, $L_{s}=108^{\circ}$ ).

profile are assumed to be constant. The following reasons allowed us to introduce the last simplification. Below we will discuss the general advantages of using the narrow wavelength range centered at $300 \mathrm{~nm}$ for the cloud retrieval. In this particular discussion it is important that the absorption cross sections of ozone and $\mathrm{CO}_{2}$ are small enough at this wavelength to allow us to neglect their temperature dependence. The role of temperature is restricted to the influence on the vertical profile of air density and hence Rayleigh scattering. The modeling had shown that variations of the surface temperature and of the temperature profile bring quite small changes to the resultant radiance factor. The temperature variations cause significant changes in the air density profile but very small ones in the total column abundance. For optically thin atmospheres, like the Martian atmosphere, the radiance factor is proportional to the total optical thickness of the atmosphere [Liou, 2002] and, hence, to the total column abundance of the air molecules.

[29] As it was shown above, the radiance factor is influenced by the ground albedo, the ozone content and by the dust and cloud optical thicknesses. The most obvious way to retrieve the cloud optical depth is to fit the radiance factor in the whole wavelength range of measurements using a forward model and varying all considered parameters. This approach faces the following difficulties. First, simultaneous retrieval of many parameters is time consuming. Although it is known from the HST measurements [e.g., Clancy et al., 1999] that the Martian surface albedo increases with the increase of wavelength, the lack of more detailed knowledge of wavelength dependences of the ground albedo and the dust optical parameters complicates the retrieval. It is undesirable to reduce the amount of data by averaging over a number of spectra because they may depend on quantities that abruptly change with the coordinates. The surface pressure varies with the relief which sometimes changes quite sharply. Also cloudiness is not always a uniform haze. There are very often compact clouds with sharp boundaries, meaning that for cloud retrieval it is better to keep the highest resolution provided by SPICAM. Secondly, the solution is not always unique. Frequently, the same value of the radiance factor can be obtained by introducing in the model a cloud or by increasing the ground albedo. The same remark is valid for dust: introducing the dust or decreasing the ground albedo gives similar results. Figure 13 illustrates the problem. The 


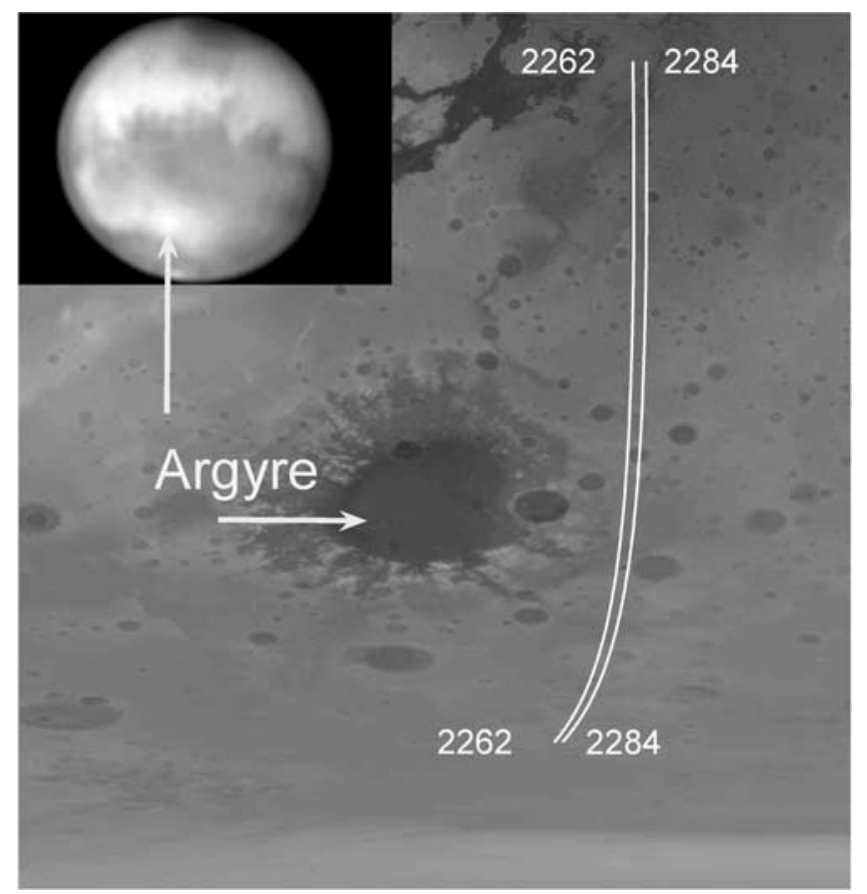

Figure 11. Parts of tracks of orbits 2262 and 2284, which passed near Argyre basin. The orbit 2284 crossed a dust storm on 24 October 2005. Inset shows a telescope image of Mars (red filter) obtained on 24 October 2005 by Joel Warren, Amarillo, TX; the dust storm is clearly visible as a white strip in the southern hemisphere (the south pole lies in the bottom of the image).

radiance factor $I / F$ versus wavelength was modeled for three different cases: without any aerosol (solid line), with dust (dashed line) and with cloud (dash-dotted line). Then both dust and ice cloud cases were fitted (crosses and triangles correspondingly) using only albedo variations.

[30] To bypass the problems mentioned above a narrow range of wavelength around $300 \mathrm{~nm}$ was considered, practically out of the ozone Hartley band. For typical values

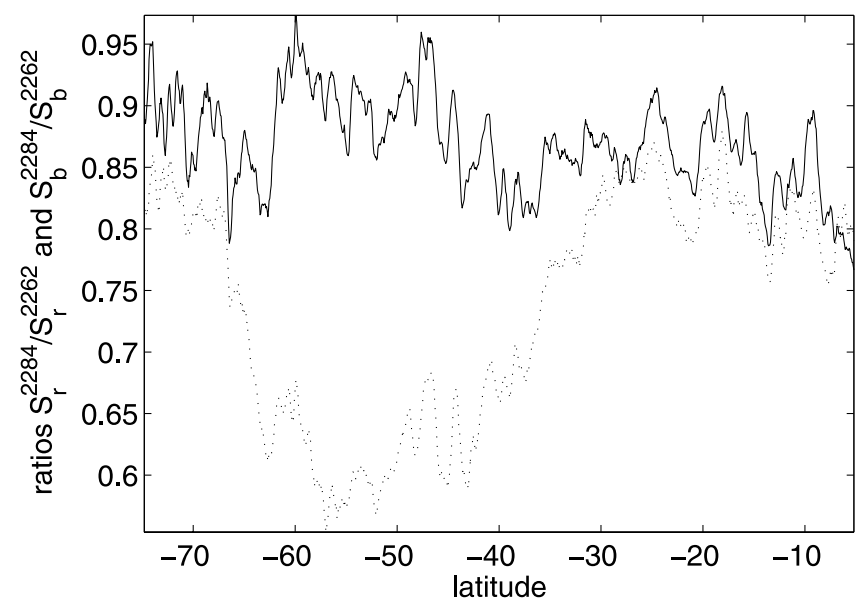

Figure 12. Ratios between the "red" SPICAM bands for orbits 2284 and 2262 (solid line) plotted in Figure 11 and the "blue" SPICAM bands for the same orbits (dotted line).

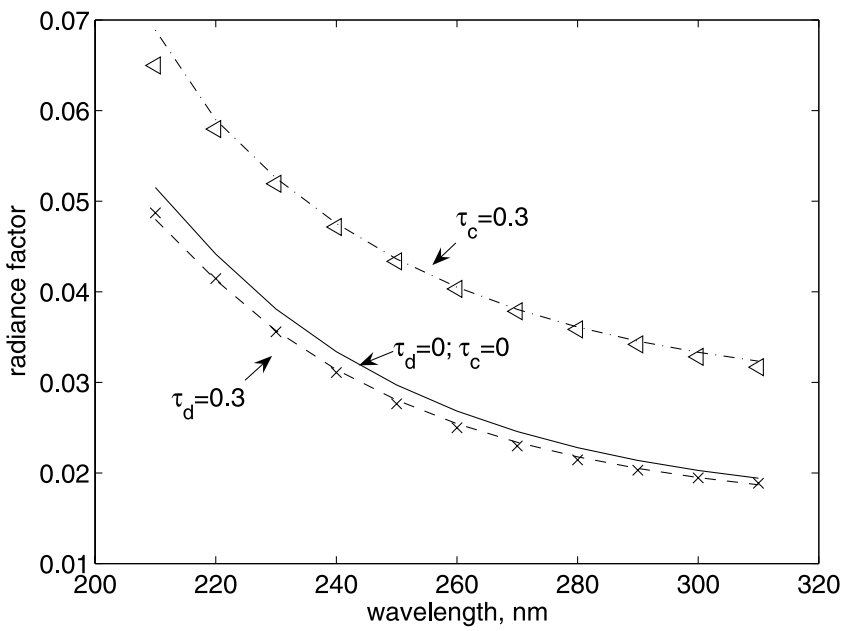

Figure 13. Modeled radiance factor in case of (1) $\tau_{d}=0$, $\tau_{c}=0$, solid curve; (2) $\tau_{d}=0.3, \tau_{c}=0$, dashed curve; and (3) $\tau_{d}=0, \tau_{c}=0.3$, dot-dashed curve. Crosses and triangles present the result of curve 2 and 3 fitting with only albedo variations.

of Martian ozone (less than $33 \mu \mathrm{m}$-atm) we can exclude ozone from our analysis (Figure 6). We have also seen in the previous section that at this particular wavelength the sensitivity of the radiance factor to dust is minimal.

[31] This allows us to conclude that for a narrow wavelength range at $300 \mathrm{~nm}$ the main parameters that influence the radiance factor are the ground albedo and the ice cloud optical depth. For further analysis we introduce a parameter that we call "apparent albedo" $A$. We try to fit the radiance factor $I / F$ at $300 \mathrm{~nm}$ by varying only the value of this "apparent albedo," which is considered as Lambertian. The quantity "apparent albedo" incorporates both variations of real albedo and its non-Lambertian features. The presence of a cloud causes a significant increase of the "apparent albedo," as well as the presence of ice.

\subsection{Opposition Effect}

[32] Before considering the presence of clouds the opposition effect should be removed. A typical dependence of apparent albedo versus phase angle $\alpha$ is presented on Figure 14. The "apparent albedo" increases with decrease of $\alpha$ showing a typical non-Lambertian behavior of the Martian soil. This is in full consistence with the Hapke theory [Hapke, 1986] of the opposition effect. The dependence $A(\alpha)$ can be fitted using the Hapke expression for a non-Lambertian albedo:

$$
A=A_{0} /(1+\operatorname{tg}(\alpha / 2) / h)
$$

where $A_{0}$ and $h$ are parameters to be fitted. Both Hapke parameters are obtained using the least squares method. The result is presented on Figure 14 (smooth solid line). The opposition effect exists for the surface but not for clouds. This is because the mutual shadowing of the particles causing the opposition effect happens only if distances between them are small enough [Hapke, 1986]. 


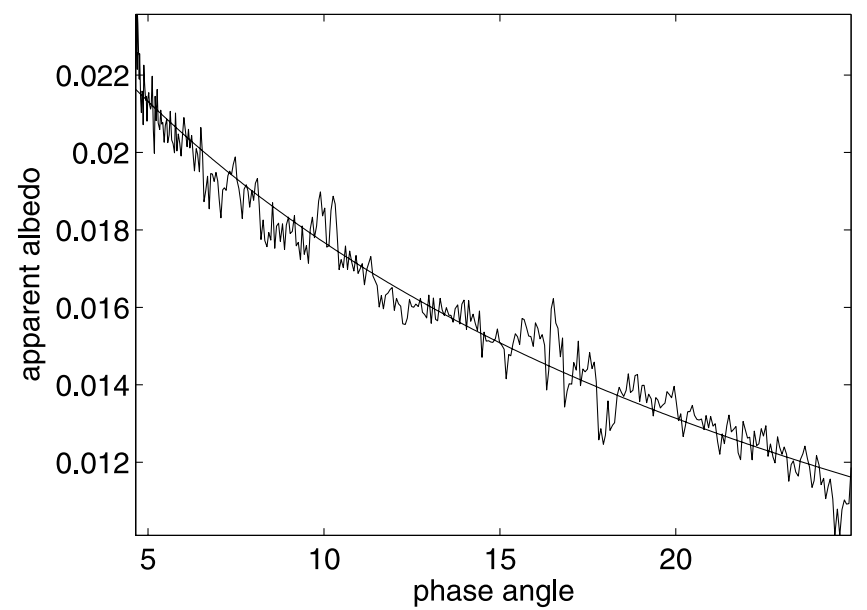

Figure 14. Non-Lambertian properties of the surface albedo (orbit 2264, wavelength $\lambda=300 \mathrm{~nm}, h=0.17$, and $\left.A_{0}=0.027\right)$.

[33] The orbits which exhibit the opposition effect, were fitted using the Hapke formula and average values of the Hapke parameters were obtained: $h=0.19, A_{0}=0.02$. These values will be used in the further analysis.

\subsection{Some Quantitative Characteristics of Dust in UV}

[34] Here we consider once more the dust storm detected at orbit 2284 by trying to obtain some quantitative characteristics of dust at wavelength $300 \mathrm{~nm}$. Pang et al. [1976] and Pang and Ajello [1977] analyzed Mariner 9 UV measurements and concluded that the dust absorption coefficient quickly decreases with the increase of the wavelength. For our analysis of ice clouds it is important that the "red" SPICAM band shows very small sensitivity to the presence of dust. The extrapolation of the spectra attributed to the orbits 2284 and 2264 (which passed almost above the same track as orbit 2284 a few days before) toward longer wavelengths indicates a minimum of sensitivity to dust at

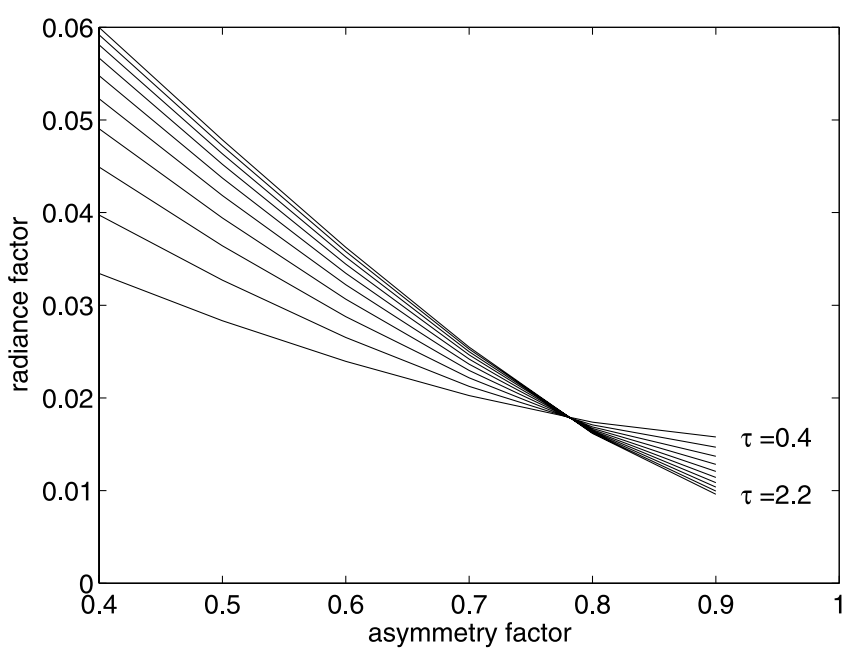

Figure 15. Radiance factor versus asymmetry factor for different dust optical thicknesses $\tau_{d}$ and for dust single scattering albedo $\sigma_{d}=0.6$ (wavelength $\lambda=315 \mathrm{~nm}$ ).

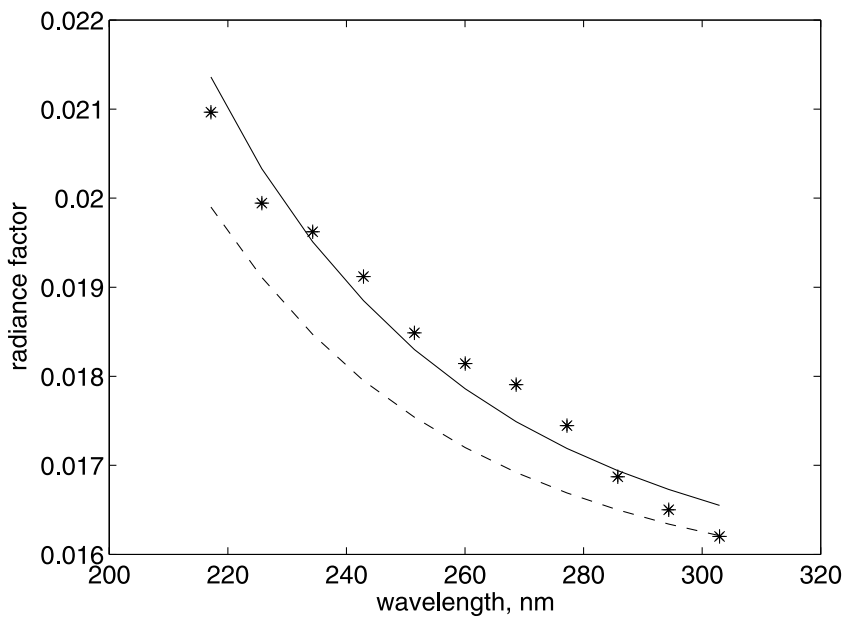

Figure 16. Spectrum acquired during the October 2005 dust storm (asterisks). The solid line indicates the result of fitting with the Conrath parameter $\gamma=0.03$, and the dashed curve is with $\gamma=0.01$.

$315 \mathrm{~nm}$. This effect is illustrated by Figure 15 where the radiance factor was modeled for wavelength $315 \mathrm{~nm}$ and is presented as a function of both dust asymmetry factor $g_{d}$ and dust optical thickness $\tau_{d}$. Exactly the same values of $S Z A$ and $\alpha$ were used as those of the dust storm detected at orbit 2287. The value of UV single scattering albedo $\sigma_{d}=$ 0.6 was adopted from Clancy et al. [1999].

[35] In general, the presence of dust causes an increase of $I / F$ at small values of the $g_{d}$ and a decrease at large ones. It is clear from Figure 15 that around $g_{d}=0.78$ the radiance factor does not depend on dust optical thickness.

[36] To adjust the value of $g_{d}$ at $300 \mathrm{~nm}$ the spectrum with expected high dust loading was chosen. The ratio of the "blue" bands of SPICAM spectra for orbits 2264 and 2284 (Figure 12) exhibits a minimum in the area of maximal dust loading. The fit of the orbit 2284 spectrum (Figure 16) acquired in this area provides the following dust optical parameters: $\tau_{d}=3.5, w_{d}=0.6, g_{d}=0.8$. The result appeared to be only slightly sensitive to the variations of the surface albedo due to high dust loading. The surface albedo was estimated by using the $300 \mathrm{~nm}$ surface albedo map (Figure 17, see section 4.5.1). The value of the surface albedo at $210 \mathrm{~nm}$ is expected to be lower than the one at $300 \mathrm{~nm}$. To represent correctly the dust extinction vertical profile the expression proposed by Conrath [1975] was used:

$$
\beta(h)=\beta_{0} \exp \left(\gamma\left(1-p_{0} / p(h)\right)\right)
$$

where $h$ is the altitude above the surface, $\beta$ is the dust extinction coefficient, $p$ is pressure, the index 0 corresponds to the values of these quantities on the surface level and $\gamma$ is a parameter. The dashed and solid curves presented at Figure 16 were modeled with different parameter $\gamma=0.01$ and 0.03 correspondingly. The value $\gamma=0.01$ is characteristic for a dust storm whereas $\gamma=0.03$ for quiet conditions. Both profiles give quite close results. The spectrum was fitted using wavelength-independent para- 


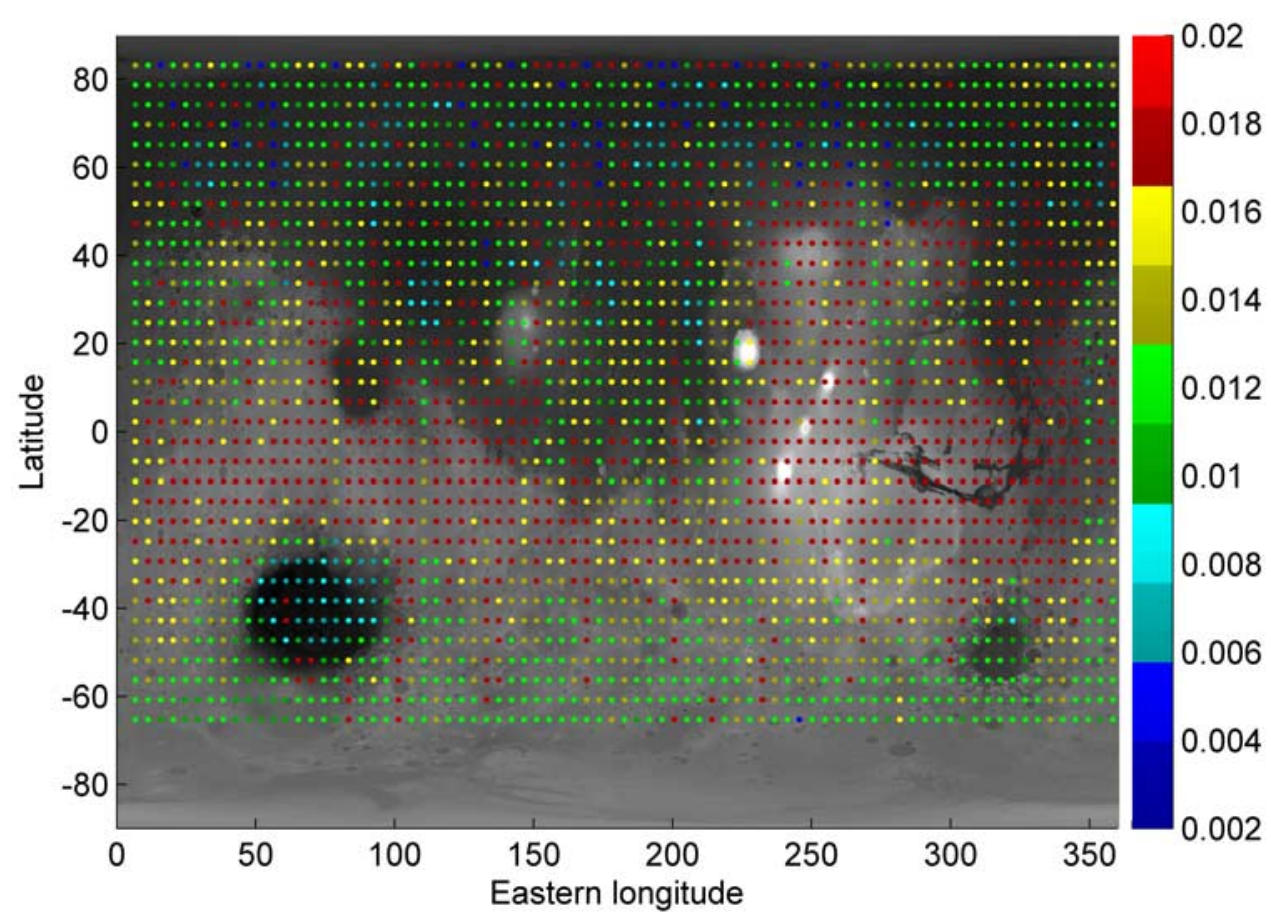

Figure 17. Ground albedo map (wavelength $\lambda=300 \mathrm{~nm}$ ) which was used as an input for cloud optical thickness retrieval.

meters $\tau_{d}, w_{d}$ and $g_{d}$, and the uniqueness of the solution was investigated.

[37] Figures $18 \mathrm{a}$ and $18 \mathrm{~b}$ show how the presence of the same amount of dust alters the radiance factor for the different values of the dust asymmetry factors $g_{d}$. The result of the modeling is shown for two values of albedo: 0.01 (Figure 18a) and 0.015 (Figure 18b). The radiance factor exhibits a moderate variation in the case of $g_{d}=0.8$ (the value obtained from the dust storm analysis). The dust effect depends on the surface albedo and the $S Z A$. The presence of dust causes the decrease of I/F at small $S Z A$ s and the effect grows with albedo increase. For $S Z A>50^{\circ}$ dust causes the increase of $\mathrm{I} / \mathrm{F}$ which is more prominent for small values of the surface albedo. The moderate variations of the $I / F$ due to presence of dust in the case of $g_{d}=0.8$ allow us to neglect of the dust effect. The uncertainty introduced by this approximation is considered in the section 4.5.4.

[38] We conclude that the estimates for dust optical properties at $300 \mathrm{~nm} \sigma_{d}=0.6$ and $g_{d}=0.8$ are reasonable.

[39] Most Martian ice clouds were observed at a period of low and moderate dust loading. This allows us to assume that the "red" SPICAM band will be minimally affected by dust and can be used for the retrieval of the ice cloud optical thickness without taking dust into account.
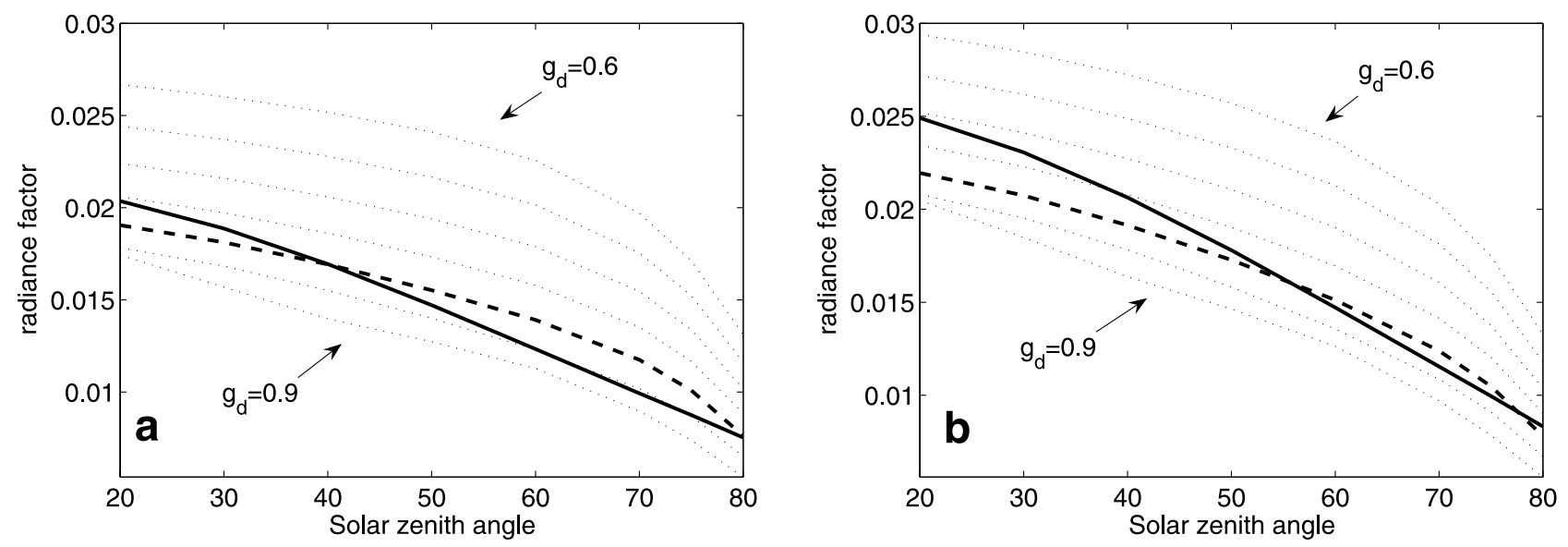

Figure 18. Radiance factor variations for dust loading $\tau_{d}=0.5, w_{d}=0.6$ and different values of $g_{d}$ (dotted lines). Solid line presents the modeled radiance factor without dust, and dashed line presents the modeled radiance factor for the adopted value of $g_{d}=0.8$. Results for surface albedo values (a) 0.01 and (b) 0.015 correspondingly. 


\subsection{Retrieval Procedure}

\subsubsection{UV Surface Albedo}

[40] The main features to be taken into account at $300 \mathrm{~nm}$ for cloud retrieval are the ground albedo and the cloud optical thickness. To discriminate the contributions of these two features we split the retrieval procedure in two steps: the construction of a surface albedo map for $300 \mathrm{~nm}$ wavelength and the retrieval of the cloud optical thicknesses using this map. In the first step the "apparent albedo" for all of the orbits is calculated. The values of "apparent albedo" are corrected for the opposition effect using Hapke formula (1) with parameters obtained as described above. After correction all "apparent albedo" values were sorted with respect to a threshold of 0.02 . This criterion was elaborated by comparison of "apparent albedos" for orbits with and without clouds, where clouds were detected from raw data (see section 3). Many clouds were quite prominent and had sharp boundaries that simplified the selection. Roughly speaking, this sorting allowed separating cloudy and clear parts of the orbits for which the "apparent albedos" coincided with the real ones.

[41] In order to build the albedo map, only values of "apparent albedo" attributed to the clear parts of orbits were used. The "apparent" albedo values $A_{\exp }$ were corrected by the opposition effect. The procedure of the correction was the following. The Hapke formula (1) with averaged parameters was used to calculate the albedo $A_{\text {theor }}$ modeled at $\alpha<$ $25^{\circ}$. The differences $A_{\text {new }}(\alpha)=A_{\text {exp }}(\alpha)-A_{\text {theor }}(\alpha)$ were calculated. The final corrected albedo values $A_{\text {corr }}$ were obtained as $A_{\text {corr }}(\alpha)=A_{\text {new }}(\alpha)-\Delta A$, where $\Delta A$ is the difference between $A_{\text {new }}$ and $A_{\exp }$ at $\alpha=25^{\circ}$. After the correction, the whole surface of Mars was divided in $4.5^{\circ}$ bins. For each bin, a histogram was build using the corrected "apparent albedo" values. The maximum of the histogram gives the most probable value for the ground albedo. The result is presented in Figure 17. The comparison between the UV albedo map and Viking visual albedo map revealed anticorrelation between them. For example, Hellas and Argyre basins appeared dark in the UV whereas Syrtis Major appeared bright (Figure 17). The visual albedo shows the opposite behavior. Bibring et al. [2006] obtained the surface mineralogy map using the measurements of the OMEGA instrument based on Mars Express. These maps show that regions with high visual albedo are abundant in ferric oxides whereas dark areas are formed by pyroxenes. The ferric oxides absorb strongly in the UV [Bell, 1996] causing the anticorrelation effect. It is worth noting that the final evaluation of the Martian ground albedo will only be possible after consideration of the opposition effect for different regions characterized by more or less uniform ground properties.

[42] Although the ice haze is mainly removed using the apparent albedo sorting procedure, it is still possible that some weak hazes introduce a bias into the surface albedo. The same happens with dust. The procedure of taking the most probable value of the "apparent albedo" in each bin in many cases allows to exclude the biases caused by strong aerosol hazes whereas the bias caused by a minimal amount of dust which always presence in the Martian atmosphere stays incorporated in the surface albedo.

\subsubsection{Cloud Optical Thickness}

[43] In the second step we consider the values of "apparent albedo" which exceed the threshold. The albedo map presented in Figure 17 was used for choosing the values of the background albedo. The following parameters were used for the ice cloud optical depth retrieval: single scattering albedo $\sigma_{c}=1$ and asymmetry factor $g_{c}=0.7$. Indeed, water ice becomes slightly absorptive in the UV. The value of $\sigma_{c}$ was estimated using the Mie theory and the optical properties of water ice at $300 \mathrm{~nm}$ taken from Warren [1984]. For ice particles, a radius value of $2 \mu \mathrm{m}$ was adopted [i.e., Clancy et al., 2003; Wolff and Clancy, 2003, and references therein]. The value of single scattering coalbedo (defined as $1-\sigma_{c}$ ) is $4 \cdot 10^{-7}$, a quite low one, and we consider the water ice absorption negligible at $300 \mathrm{~nm}$. The water ice cloud asymmetry factor $g_{c}$ is not well known in the UV. Clancy and Lee [1991] estimated $g_{c}=0.66$ using emissionphase-function observations from the Viking IRTM broadband $(0.3-3.0 \mu \mathrm{m})$ visible channel centered at $0.67 \mu \mathrm{m}$. On the other hand parameterized properties of the Earth's cirrus clouds give a value of 0.75 [Key et al., 2002], consistent with solid columns of effective radius $6 \mu \mathrm{m}$ at $300 \mathrm{~nm}$ wavelength. We believe that the value $g_{c}=0.7$ is a reasonable compromise between the two estimates. We adopted cloud altitude of $15 \mathrm{~km}$ for the ACD and $25 \mathrm{~km}$ for the other clouds with assumed $1 \mathrm{~km}$ cloud thickness.

[44] An important issue to be considered is the boundary of seasonal ices. We cannot distinguish between ices and clouds only by using their UV spectra. Fortunately, the retreat of the north seasonal polar cap is well documented by both TES [Titus, 2005] and MOC [Benson and James, 2005] measurements. These data were used to determine the position of the north polar cap edge versus $L_{s}$. To be sure that we do not observe ices, we widened this edge by $2^{\circ}$. For the correct detection of the north summer clouds, the MOC atlas was used. A more complex situation had to be considered in the south pole region. For $L_{s}$ range $159-245^{\circ}$ TES data [Christensen et al., 2001] were used with a $2^{\circ}$ offset. During other periods, we considered as valid south polar hood clouds only the ones having an apparent albedo lower than 0.05 , a value significantly lower than the southern ice albedo.

[45] Examples of the retrieved cloud optical thicknesses are shown in Figures $19 \mathrm{a}-19 \mathrm{~d}$. The retrieval procedure is summarized on Figure 20.

\subsubsection{Cloud Optical Thickness Spectral Behavior}

[46] To retrieve the cloud optical thickness for the whole spectral range of measurements $(210-308 \mathrm{~nm})$ it is necessary to know the spectrally dependent surface albedo and the dust optical thickness. The problem can be simplified if we consider the dense cloud detected at orbit 1177 above Olympus Mons (Figures 7 and 8). The high altitude of the volcano and the period of observations $\left(L_{s}=131^{\circ}\right)$ allows to suppose low dust loading. To estimate the surface albedo, the cloudless orbit 1448 which passed very close to the track of the orbit 1177, was used. Figure 21 presents the fitting result of two spectra from orbit 1177 and 1448 acquired above almost the same area. The surface albedo retrieved from the spectrum of orbit 1448 was used to retrieve the cloud optical thickness from the spectrum of 

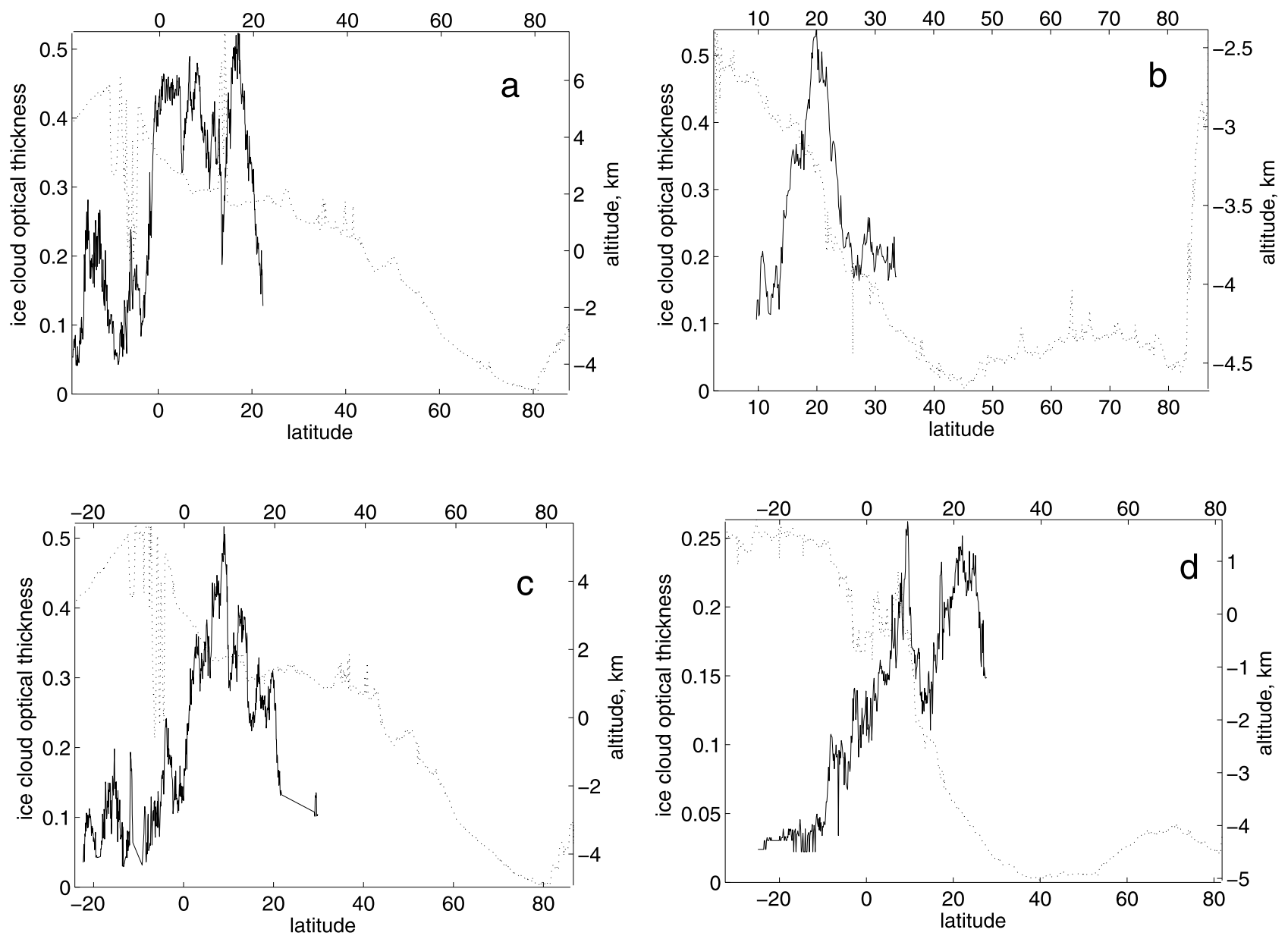

Figure 19. Cloud optical thicknesses (solid line) observed at orbits (a)1041 $\left(L_{s}=113^{\circ}\right)$, (b) $1090\left(L_{s}=119^{\circ}\right)$, (c) $1008\left(L_{s}=109^{\circ}\right)$, and (d) $962\left(L_{s}=103^{\circ}\right)$ and surface altitude (dotted line).

orbit 1177 . The spectrally independent cloud optical thickness appeared to allow for a good fit. The result is not surprising if we remember that the ice cloud particles are quite large $(2-4 \mu \mathrm{m}$ [Clancy et al., 2003]) with respect to the wavelength 200-300 $\mathrm{nm}$ whereas water ice absorption is very weak in this wavelength domain [Warren, 1984].

\subsubsection{Uncertainties}

[47] The uncertainties can be divided on two categories: the measurement uncertainties and the modeling uncertainties.

[48] The SPICAM calibration issue was discussed in details by Bertaux et al. [2006]. Here we only consider the random noise error. As we do not have multiple measurements in one point, we should distinguish between the noise and natural signal variability induced by the variations of the surface albedo and pressure along the track. The parts of the orbits where the averaged signals $S_{r}$ and $S_{b}$ shows zero trends with respect to coordinates and an uniform level of noise were used. The error was estimated to be about $1 \%$ of the signal magnitude. The error connected with the signal random variations with wavelength within the "red" and "blue" SPICAM bands was estimated as about $4 \%$. The noise can be reduced by averaging the retrieved optical thickness values and does not play an important role in the total error budget.
[49] The much more important sources of error are uncertainties connected with the forward model through assumed simplifications and uncertainties of the adopted optical parameters.

[50] Hereafter we consider the following parameters and assumptions as sources of uncertainties: the cloud altitudes and the thickness of the layer, the assumption that dust can be neglected, the water ice aerosol optical properties, and the surface albedo.

[51] Many of the considered uncertainties appeared to be a function of the solar zenith angle (Figure 22 presents the $S Z A$ s distribution for the detected clouds). The altitude of the Martian ice clouds vary between $h_{c}=10-15 \mathrm{~km}$ in the period of the ACB formation and $25-30 \mathrm{~km}$ in other periods. These estimates come from the modeling of the hydropause altitude [Richardson et al., 2002; Smith, 2002] and from measurements [Jaquin et al., 1986; Rodin et al., 1997; Pearl et al., 2001]. To estimate the uncertainty due to the cloud altitude variation, the cloud optical thicknesses were retrieved for the two values of $h_{c}=15$ and $25 \mathrm{~km}$ with a $1 \mathrm{~km}$ cloud thickness. The difference between both data sets appeared $2-3 \%$ for the $S Z A$ s less than $75^{\circ}$ but quickly grows with the increase of the $S Z A$ above this value. The increase of the cloud thickness from $1 \mathrm{~km}$ to $5 \mathrm{~km}$ gives the $3 \%$ uncertainty for the $S Z A$ s less than $75^{\circ}$. The dustless 


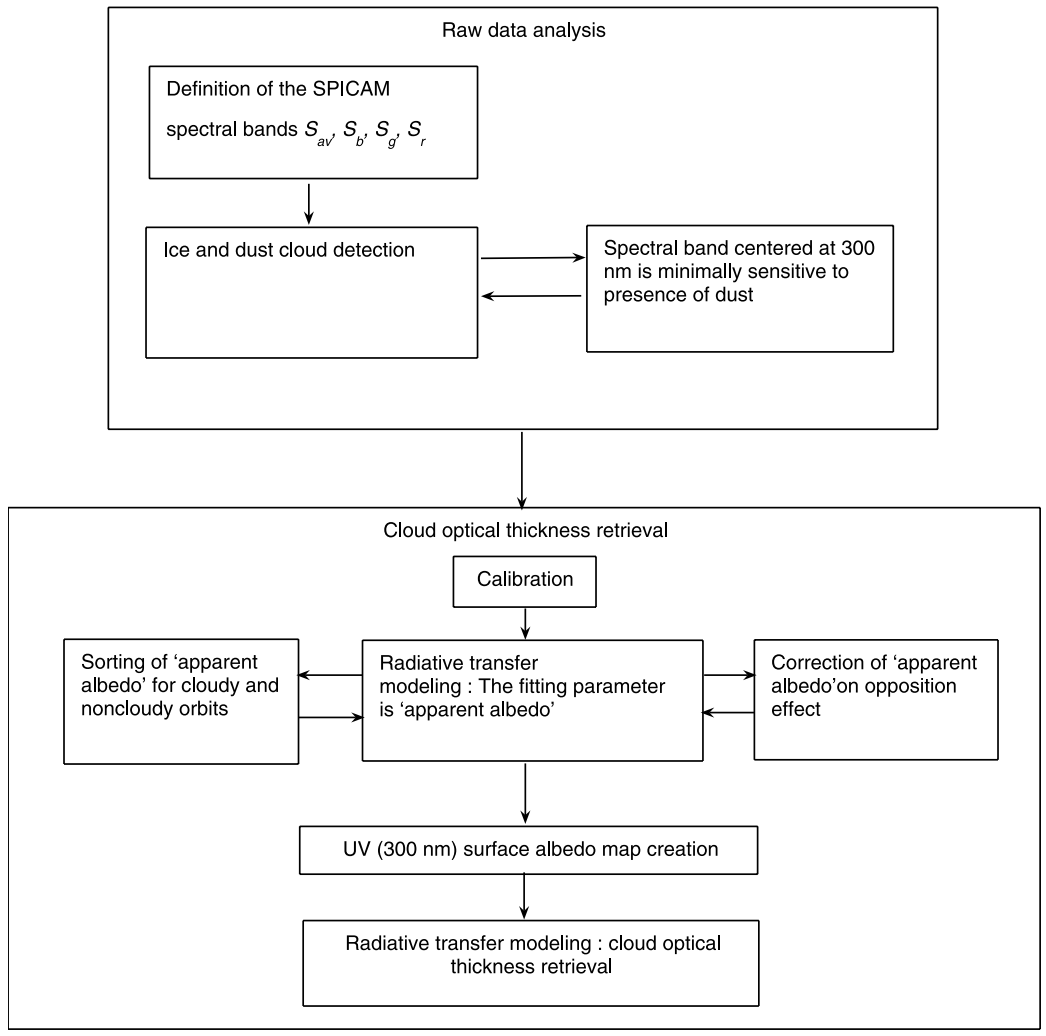

Figure 20. A scheme of the cloud optical thickness retrieval procedure.

atmosphere is optically thin and the observation is mainly dominated by the total cloud optical thickness rather than by the exact extinction vertical profile.

[52] To estimate the uncertainties due to neglecting of dust, the modeling were performed for the following set of parameters. Two values of the surface pressure 6 and 9 mbar were considered. The surface albedo varied from 0.005 to 0.018 , covering the observed range of albedo. The $S Z A$

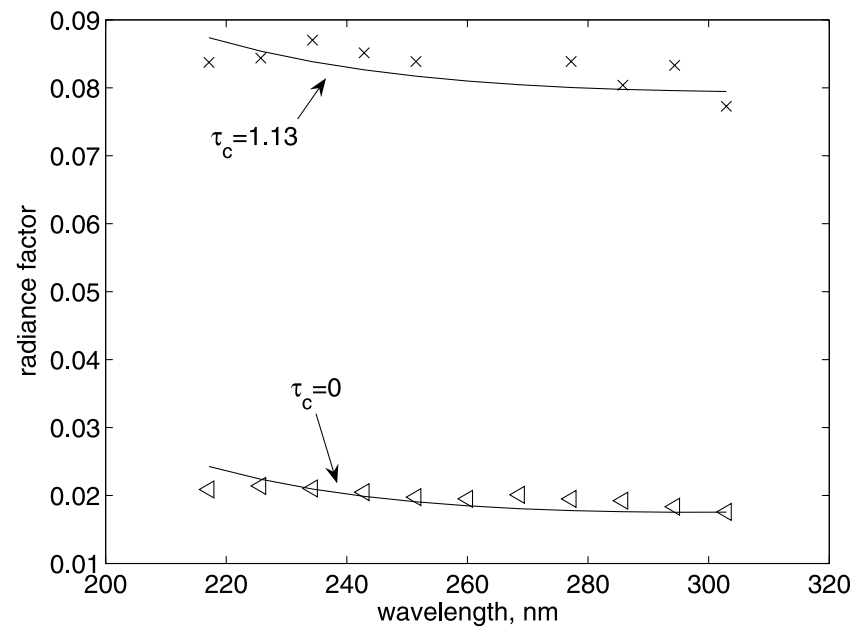

Figure 21. Result of fitting (solid curves) for two spectra from orbit 1177 (crosses) and 1448 (triangles) acquired above almost the same area. The surface albedo retrieved from the spectrum of orbit 1448 was used to retrieve the cloud optical thickness from the spectrum of orbit 1177. varied from $20^{\circ}$ to $80^{\circ}$. Two cases of cloud optical thickness $\tau_{c}=0.3$ and 0.5 lofted at 15 and $25 \mathrm{~km}$ were considered. The dust optical thickness $\tau_{d}=0.3$ were adopted as a characteristic dust opacity for the aphelion period that is consistent with both HST results [Wolff et al., 1999] and estimates made by Perrier et al. [2006] using SPICAM data. Figure 23 shows the result of modeling for $\tau_{c}=0.5$ lofted at $15 \mathrm{~km}$ and two values of albedo. The curves

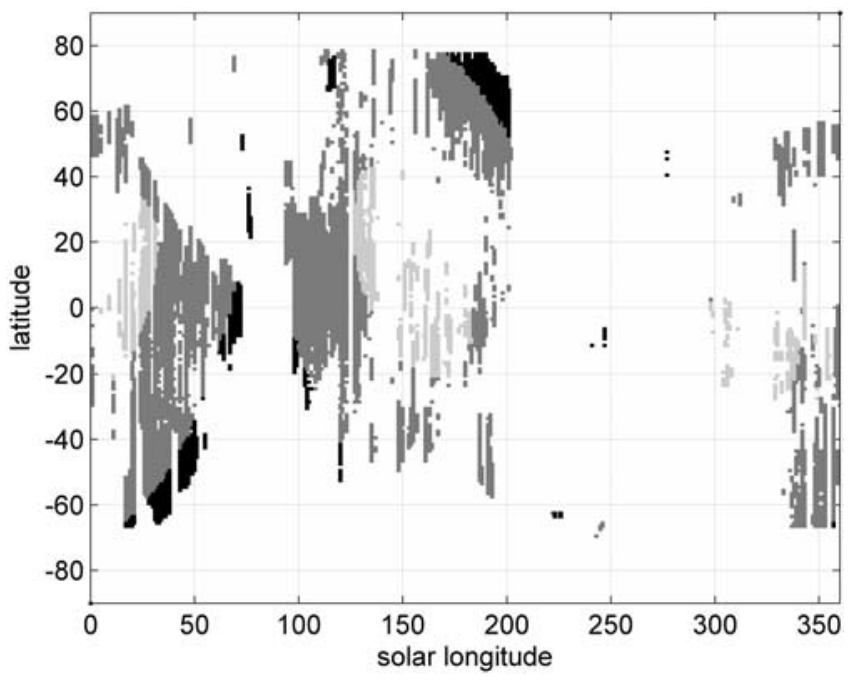

Figure 22. Solar zenith angles distribution for the cloud data set. Light shading indicates $S Z A<30^{\circ}$, darker shading indicates $S Z A>30^{\circ}$ and $<75^{\circ}$, and solid indicates $S Z A>$ $75^{\circ}$. 

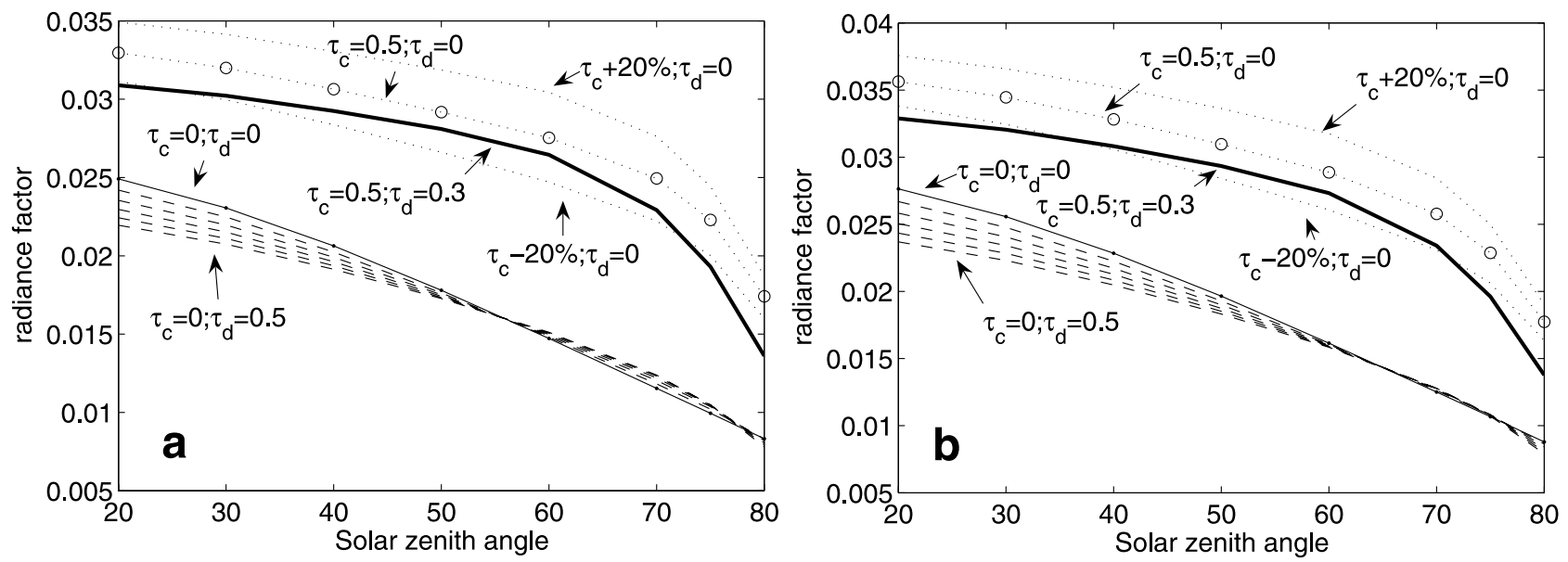

Figure 23. Modeling of uncertainties due to dust neglecting. (a) Surface albedo $\mathrm{A}=0.01$ and (b) surface albedo $\mathrm{A}=0.015$. The result of modeling for $\tau_{c}=0.5$ lofted at $15 \mathrm{~km}$. Solid curve indicates $I / F$ factor in absence of both dust and ice clouds, dashed curves indicate $I / F$ modeled with $\tau_{d}$ varying from 0.1 to 0.5 , dotted line with circles indicates $I / F$ in presence of an ice cloud without dust, and two dotted lines indicate the $I / F$ modeled with $\tau_{c} \pm 20 \%$. The bold solid curve shows $I / F$ in case of presence both ice cloud and dust.

presented in Figure 23 are the radiance factor $I / F$ versus $S Z A$ in absence of both dust and ice clouds (solid curve), $I / F$ for different amount of dust loading (dashed curves), $I / F$ in presence of an ice cloud without dust (dotted line with circles) and two dotted lines representing the effect of a $20 \%$ variation of $\tau_{c}$. The bold solid curve shows the $I / F$ factor in case of presence both ice cloud and dust. The difference between the case of ice cloud without dust and the case of ice cloud with dust suggests the uncertainties of modeling. The uncertainty depends on $S Z A$ and is typically about $20-$ $25 \%$ for $S Z A<30^{\circ}$ and $10-15 \%$ for $S Z A>30^{\circ}$ but quickly increases with $S Z A$ for $S Z A$ s larger than $75^{\circ}$. As observed in Figures $23 \mathrm{a}$ and $23 \mathrm{~b}$, the uncertainty grows with albedo. It should be mentioned that the uncertainty caused by neglecting of dust may be slightly lower than the estimate given above because the used albedo value includes some minimal dust loading (section 4.5.1).

[53] The error estimation for the cloud with $\tau_{c}=0.3$ lofted at $25 \mathrm{~km}$, a typical value for clouds outside the ACB, gives a similar result as for $\tau_{c}=0.5$. The uncertainty increases with cloud altitude decreasing. It is about $30 \%$ for $S Z A<30^{\circ}$ and $20 \%$ for $S Z A>30^{\circ}$ when a cloud with $\tau_{c}=0.3$ is lofted at $15 \mathrm{~km}$. The variations of the surface pressure do not influence on the results.

[54] Another important source of cloud optical thickness uncertainty results from the surface albedo knowledge, estimated as the most probable value of the apparent albedo in $4.5^{\circ}$ coordinate bins. The albedo uncertainty for a particular bin consists of variations along the bin and between the different orbits. The first one is typically 5-
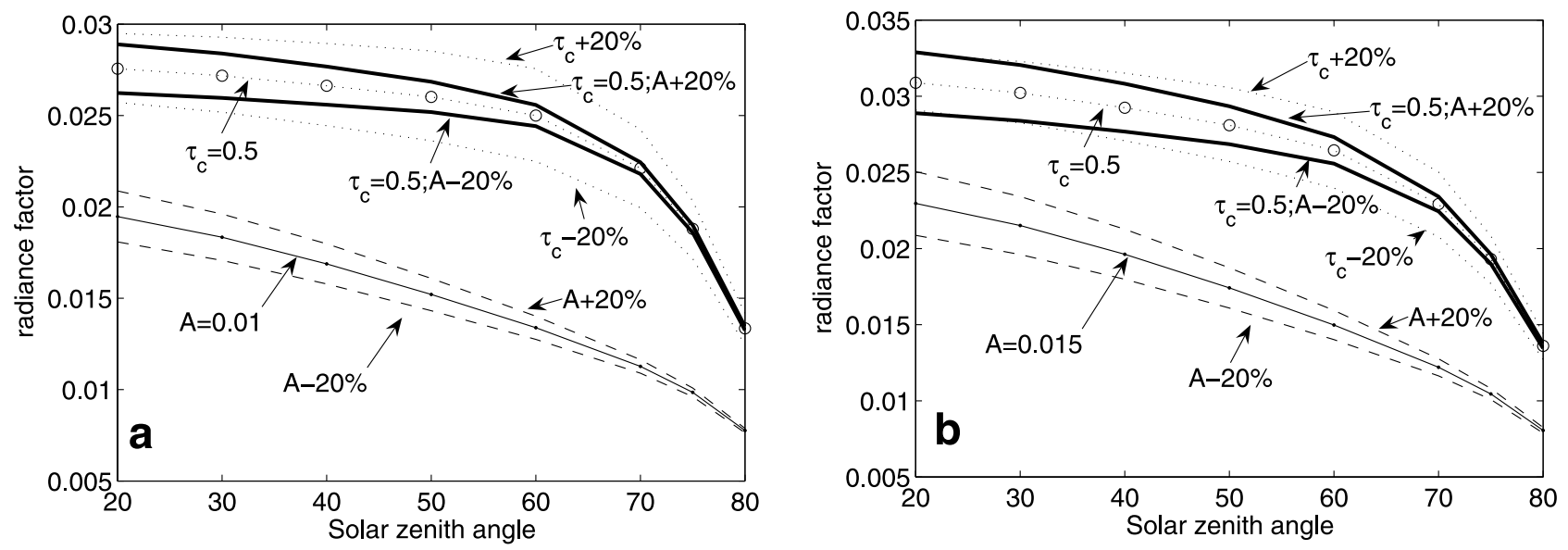

Figure 24. Modeling of uncertainties due to surface albedo error. (a) Surface albedo $A=0.01$ and (b) surface albedo $\mathrm{A}=0.015$. The modeling was performed in presence of dust with $\tau_{d}=0.3$. Solid line indicates $I / F$ with dust but without cloud. Two dashed lines indicate $I / F$ with albedo uncertainty $\pm 20 \%$. Dotted line with circles indicates $I / F$ in presence of a cloud with $\tau_{c}=0.5$ lofted at $15 \mathrm{~km}$. Dotted lines indicate $I / F$ for $\tau_{c} \pm 20 \%$, and bold solid lines indicate $I / F$ in presence of a cloud and with albedo uncertainty $\pm 20 \%$. 


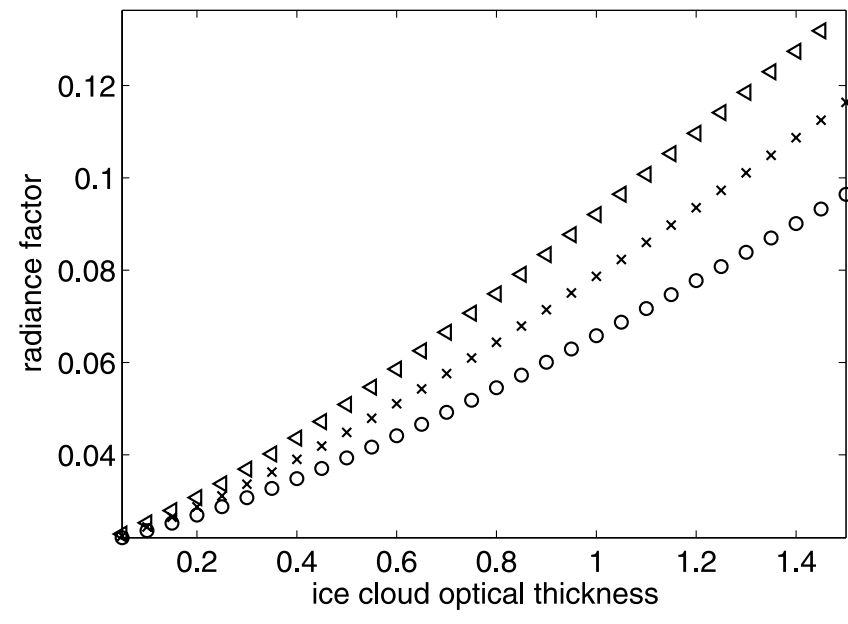

Figure 25. Radiance factor $\mathrm{I} / \mathrm{F}$ dependence on to the ice cloud asymmetry factor variations. Triangles indicate $g_{c}=$ 0.65 , crosses indicate $g_{c}=0.70$, and circles indicate $g_{c}=$ 0.75 .

$10 \%$ whereas the total uncertainty is about $20 \%$ and reaches $40 \%$ for low values of the surface albedo, indicating the influence of aerosol hazes when incorporated in the "apparent" albedo values.

[55] The cloud optical thickness uncertainty caused by the surface albedo error was investigated for the same set of parameters as the uncertainties caused by dust. The modeling shows an uncertainty decrease in presence of dust. Therefore, to have a realistic impression about the total error caused by both dust and albedo, the albedo uncertainties are considered in presence of dust. Figure 24 shows the result of modeling a cloud with $\tau_{c}=0.5$ lofted at $15 \mathrm{~km}$ in presence of dust with $\tau_{d}=0.3$ for two representative values of albedo. The solid line shows $\mathrm{I} / \mathrm{F}$ factor with dust but without cloud and two dashed lines show how $I / F$ perturbs in case of $\pm 20 \%$ change of the surface albedo. The dotted line with circles shows $I / F$ in presence of a cloud and two dotted lines present the change of $I / F$ in case of $\pm 20 \%$ change of $\tau_{c}$. The two bold solid lines show the influence of the albedo uncertainty on $I / F$ in presence of a cloud. The uncertainty increases with albedo increase and with decrease of the $S Z A$. It is typically about $20 \%$ for $S Z A<$ $30^{\circ}$ and $10-15 \%$ for $S Z A>30^{\circ}$ and quickly decreases with $S Z A$ for $S Z A$ s larger than $70^{\circ}$.

[56] We conclude that both dust and albedo errors cause the following uncertainties: $40 \%$ for $S Z A<30^{\circ}, 20-30 \%$ for $S Z A>30^{\circ}$ and up to $50 \%$ for $S Z A>75^{\circ}$.

[57] The uncertainty in the ice cloud asymmetry parameter $g_{c}$ brings a systematic shift in the entire cloud data set. Figure 25 shows $\mathrm{I} / \mathrm{F}$ versus cloud optical thickness for $g_{c}=$ $0.7 \pm 0.05$. In order to realize an influence of the uncertainty of $g_{c}$ on the estimated cloud optical thickness $\tau_{c}$ we consider the similarity relation $f=\tau_{c} \sigma_{c}\left(1-g_{c}\right)$ that was introduced for a correct estimation of the forward scattering peak [Liou, 2002]. As ice cloud particles are large in comparison with the wavelength, they should have a highly peaked phase function in the forward direction. The similarity relation can be used to estimate the uncertainty that arises from the uncertain optical parameters of cloud ice particles. Figure 26 shows how the value of radiance factor $I / F$ depends on $f$ for different $g_{c}=0.5-0.8$ and $\tau_{c}=0.1-2$ ( $\sigma_{c}=1$ for ice particles). We can see that, at a fixed $f, I / F$ is almost insensitive to $g_{c}$ and $\tau_{c}$ alterations. This dependence can be roughly approximated by an expression $\tau_{c}\left(1-g_{c}\right)=$ const, i.e., if $\tau_{c}$ is retrieved for some value of $g_{c}$, it is possible to estimate $\tau_{c}$ for a different value of $g_{c}$. The similarity relation gives about $17 \%$ uncertainty for the entire data set in case of $g_{c}=0.7 \pm 0.05$. The similarity relation also can be used for comparison with results of other works where a different value of $g_{c}$ was used. The uncertainty of such a rescaling is about $2-5 \%$ for $g_{c}=0.7 \pm 0.05$ and $S Z A<75^{\circ}$

\section{Cloud Distribution}

\subsection{General Description}

[58] In this section we describe the cloud distribution observed by SPICAM starting from spring equinox $\left(L_{s}=\right.$ $\left.0^{\circ}\right)$. Figure 27 presents zonal means of the cloud optical thicknesses. The data were averaged over $1^{\circ}$ of latitude by $1^{\circ}$ of solar longitude bins. The ACB was the most prominent feature in the northern low latitudes. It developed during northern spring, starting around $L_{s}=20^{\circ}$ and stayed well prominent during the first half of the northern summer $\left(L_{s}=90-140^{\circ}\right)$. The gap in the observations between $L_{s}=$ $77^{\circ}$ and $93^{\circ}$ and insufficient orbital coverage did not allow us to estimate the solar longitude where the zonal mean optical thickness of the ACB clouds reached its maximum. The belt decayed quickly after $L_{s}=140^{\circ}$. This general evolution of the ACB was very similar to that described by Wang and Ingersoll [2002] by analyzing the MOC images and by Smith [2004] considering TES measurements.

[59] Apart from the ACB, a small separate group of clouds was observed during the northern summer $\left(L_{s}=\right.$ $90-140^{\circ}$ ) in the vicinity of the north pole. The north polar summer clouds (NPSC) were also observed by MOC [Cantor et al., 2002; Wang and Ingersoll, 2002] and TES [Tamppari et al., 2006]. The NPSC often have a spiral shape

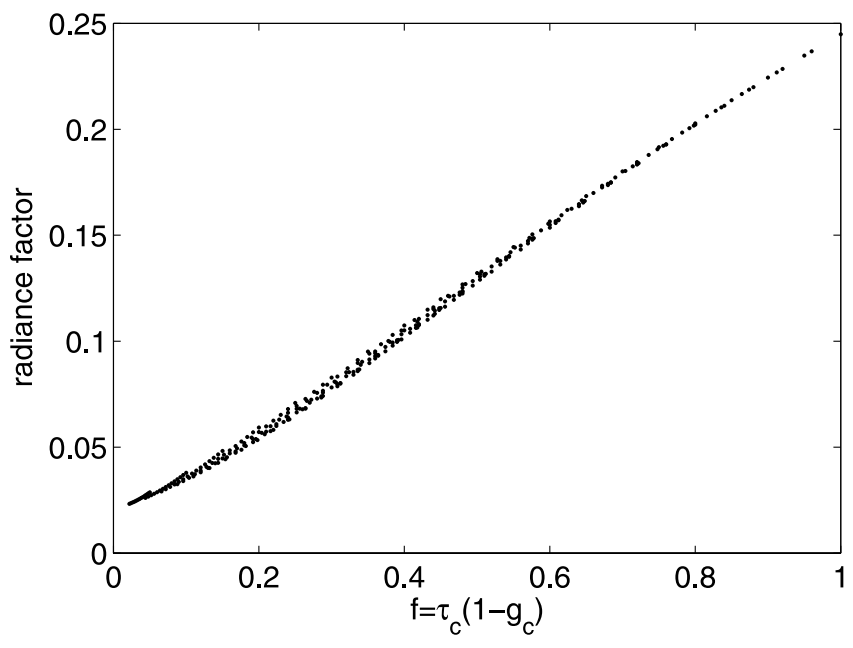

Figure 26. Dependence of the radiance factor from the combination of ice cloud optical parameters $f=\tau_{c}\left(1-g_{c}\right)$. 


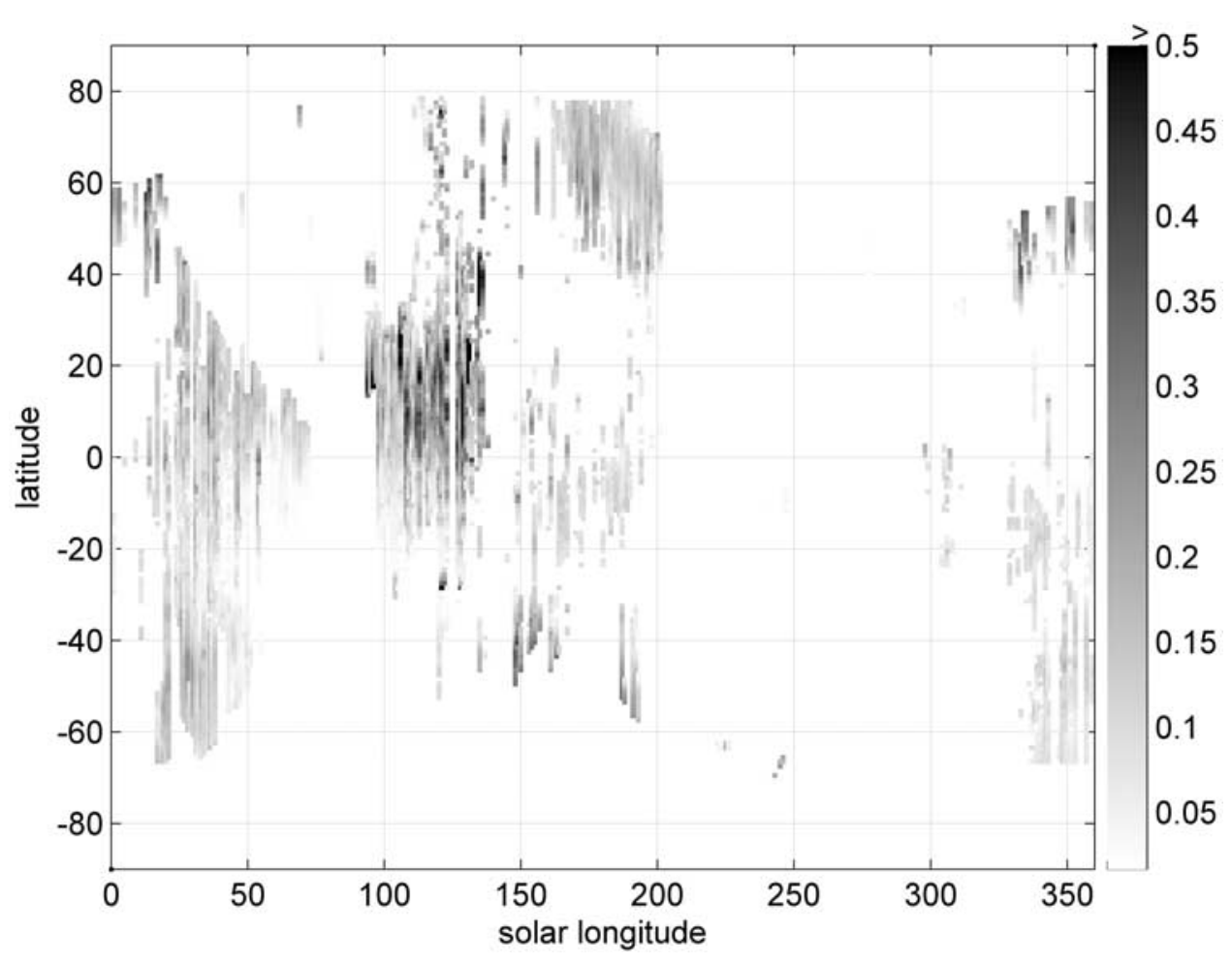

Figure 27. Zonally averaged cloud optical thicknesses versus $\mathrm{L}_{\mathrm{s}}$.

and are accompanied by dust storms [Cantor et al., 2002, Wang and Ingersoll, 2002].

[60] The only clouds observed in the tropical region during the southern summer (around $L_{S}=300^{\circ}$ ) were tiny clouds above Arsia Mons and Pavonis Mons. Benson et al. [2003] registered cloud activity above Arsia Mons in the same period, analyzing MOC images.

[61] The north polar hood started to develop in the end of northern summer $\left(L_{s}=160^{\circ}\right)$ and expanded southward till $40^{\circ} \mathrm{N}$ in the very beginning of northern fall $\left(L_{s}=200^{\circ}\right)$. Wang and Ingersoll [2002] and Pearl et al. [2001] reported the same period of polar hood formation. Tamppari et al. [2000] registered the boundary of the north polar hood at latitude $40^{\circ} \mathrm{N}$ at $L_{s}=200^{\circ}$. The gap in observations $\left(L_{s}=\right.$ $200-240^{\circ}$ ) and the latitudinal range of orbits allowed to register the north polar hood again only in the end of northern winter $\left(L_{s}=320^{\circ}\right)$. The retreat of the north polar hood was observed till the beginning of spring $\left(L_{s}=30^{\circ}\right)$.

[62] The formation of the south polar hood started in the end of southern summer, about $L_{s}=330^{\circ}$. The south polar hood grew northward till the middle of the southern winter $\left(L_{s}=150^{\circ}\right)$ and then started to retreat. The same dynamics of the south polar hood development was observed by Wang and Ingersoll [2002].

[63] As clouds exhibit diurnal variations [e.g., Wolff et al., 1999; M. D. Smith et al., 2003], the map of the local time distribution through the cloud data set is provided (Figure 28). It is possible to see that all clouds attributed to ACB were observed essentially afternoon. According to M. D. Smith et al. [2003] the cloud optical thickness increases in the late afternoon.

\subsection{Detailed Cloud Distribution}

\subsubsection{Northern Winter and Spring}

[64] The most prominent observed cloud system in the end of northern winter-beginning of northern spring $\left(L_{s}=\right.$ $330-20^{\circ}$ ) was the north polar hood (Figure 27). The optical thicknesses of the polar hood clouds were retrieved only for those parts of the orbits that passed above the bare ground because of the problems of separation of the cloud effect from the bright ice albedo. As the spring progressed and the polar hood became thinner, it was possible to distinguish the seasonal ice boundary. Northern ices manifest themselves as

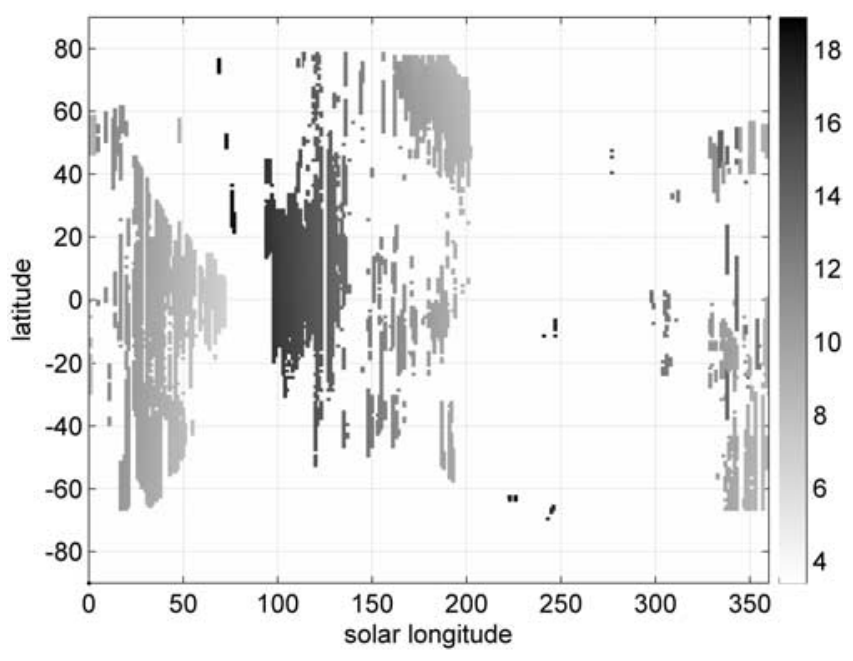

Figure 28. Local time distribution for the cloud data set. 


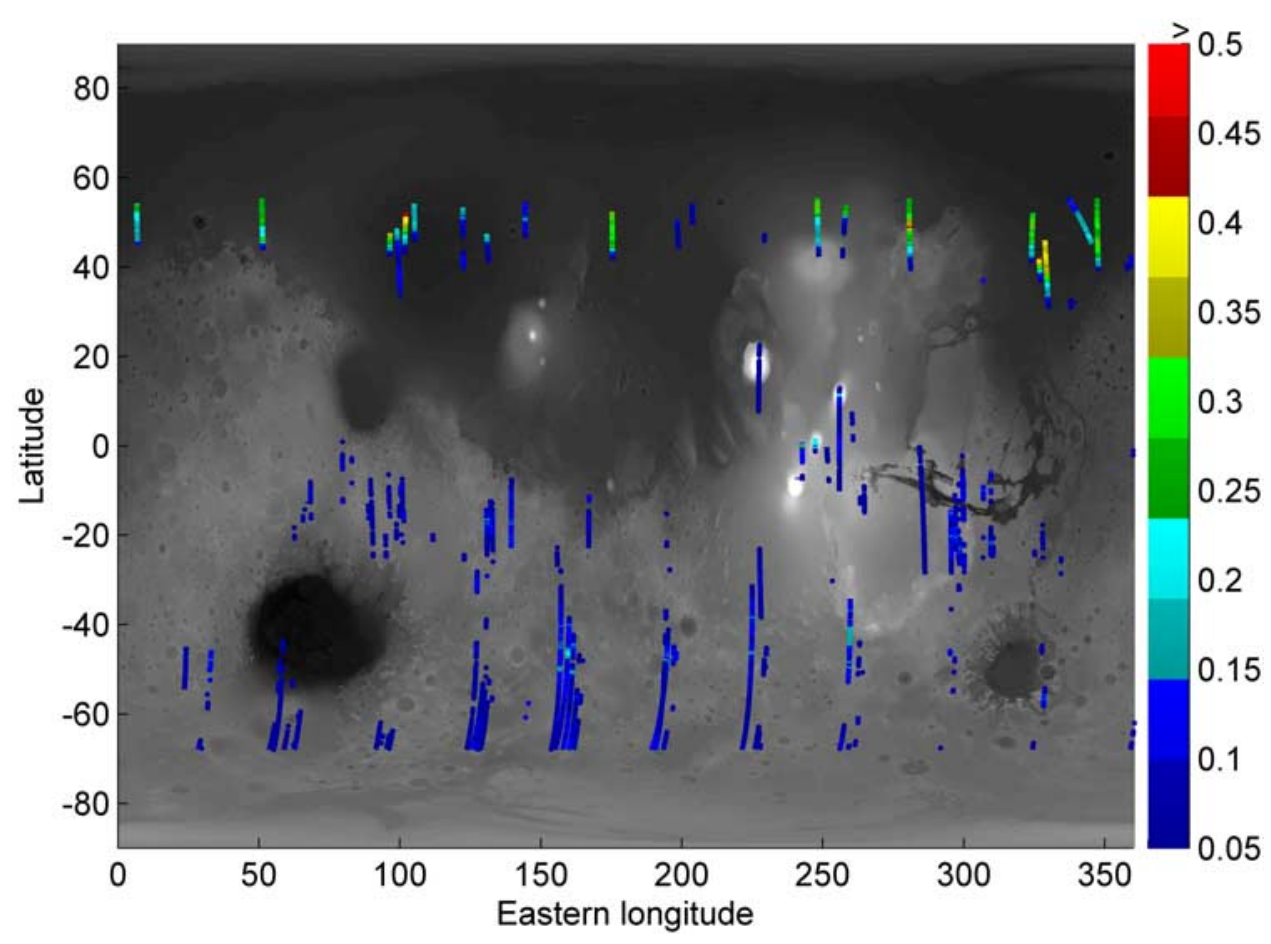

Figure 29. Cloudiness for $\mathrm{L}_{\mathrm{s}}=290-360^{\circ}$.

a sharp increase of the value of "apparent" albedo. The ice boundary was clearly observed at $60-62^{\circ} \mathrm{N}\left(L_{s}=0-20^{\circ}\right)$ in agreement with the TES [Titus, 2005] and MOC [Benson and James, 2005] measurements. The polar hood retreated from $31-47^{\circ} \mathrm{N}\left(L_{s}=340-350^{\circ}\right)$ to $45-50^{\circ} \mathrm{N}\left(L_{s}=10-\right.$ $20^{\circ}$ ). The hood south boundary slightly varied with longitude penetrating deeper southward in Acidalia Planitia up to Chryse Planitia and in Utopia Planitia. Wang and Ingersoll [2002] also mentioned this longitudinal asymmetry by analyzing MOC images. Viking observed the north polar hood penetrating deeper southward in the $180-360^{\circ} \mathrm{E}$ longitude range at $L_{s}=5-20^{\circ}$ [Tamppari et al., 2000].

[65] Separate clouds appeared in the Tharsis region and in the vicinity of Alba Patera in the considered time period $\left(L_{s}=330-20^{\circ}\right)$.

[66] Starting from the end of southern summer $\left(L_{s}=340^{\circ}\right.$, Figure 29), clouds appear in the south hemisphere in the zonal belt $30-60^{\circ} \mathrm{S}$, mainly between $120^{\circ} \mathrm{E}$ and $270^{\circ} \mathrm{E}$ indicating a beginning stage of the south hood formation. The cloud optical thickness reached its maximal value of about 0.2 around $55^{\circ} \mathrm{S}$. Wang and Ingersoll [2002] observed the first appearance of the south polar hood clouds at $L_{s}=$ $340^{\circ}$. Tamppari et al. [2000] also observed a cloud band centered at about $50^{\circ} \mathrm{S}$ in the same period. TES measurements [Smith, 2004] showed the beginning of the south polar hood formation to be different in different years. In MY 25 it happened at about $L_{s}=0^{\circ}$ whereas in MY 26 it was at about $L_{s}=340^{\circ}$.

[67] As spring progressed $\left(L_{s}=20-40^{\circ}\right.$, Figure 30) cloudiness in the tropics grew to the east of the Tharsis region and above Lunae Planum. Weak orographic clouds were visible above Elysium Mons, Ascreas Mons and Alba Patera. A group of clouds appear above Syrtis Major indicating the intensive development of the ACB. Both
Wang and Ingersoll [2002] and Tamppari et al. [2000] observed clouds in the Tharsis region during this period, but other parts of the ACB appeared in their measurements later, after $L_{s}=44-50^{\circ}$.

[68] In the same period $\left(L_{s}=20-40^{\circ}\right)$, clouds became more prominent in the southern hemisphere. The maximal cloud optical thickness was centered at $50^{\circ} \mathrm{S}$ forming a circumpolar ring (Figure 30). Both MOC [Wang and Ingersoll, 2002] and TES [Smith, 2004] observed the edge of the growing south polar hood in the same period whereas this south midlatitude belt was visible prior to $L_{s}=30^{\circ}$ according to Viking measurements [Tamppari et al., 2000].

[69] An interesting feature on the Figure 30 is a haze "bridge" (longitude range $250-340^{\circ} \mathrm{E}$ ) between the south polar hood and the ACB (Figures 27 and 30), which is most intense above Solis Lacus. The "bridge" clouds had low optical thickness $(0.07-0.1)$. This "bridge" actually should include also the north polar hood, which extends essentially southward for the considered period but the orbital coverage did not allow to observe it. A very similar feature was observed by TES in the same period [Smith, 2004]. Figure 31 presents MOC blue and red images taken at $\mathrm{L}_{\mathrm{s}}=30^{\circ}$, MY 27 (the year of SPICAM measurements), where it is possible to see this slight haze extending from the south to the north. In the same time the distribution of water vapor showed minimal values around the south pole [Fedorova et al., 2006]. This may indicate that ice particles of the south polar hood are rather transported from the north and not formed in situ.

[70] The ACB continued to develop in the late spring between $L_{s}=40^{\circ}$ and $L_{s}=77^{\circ}$. Clouds were observed above Lunae Planum, at the east of the Tharsis region, at Chryse Planitia and Syrtis Major. The haze "bridge" between the tropical region and the south polar hood became less 


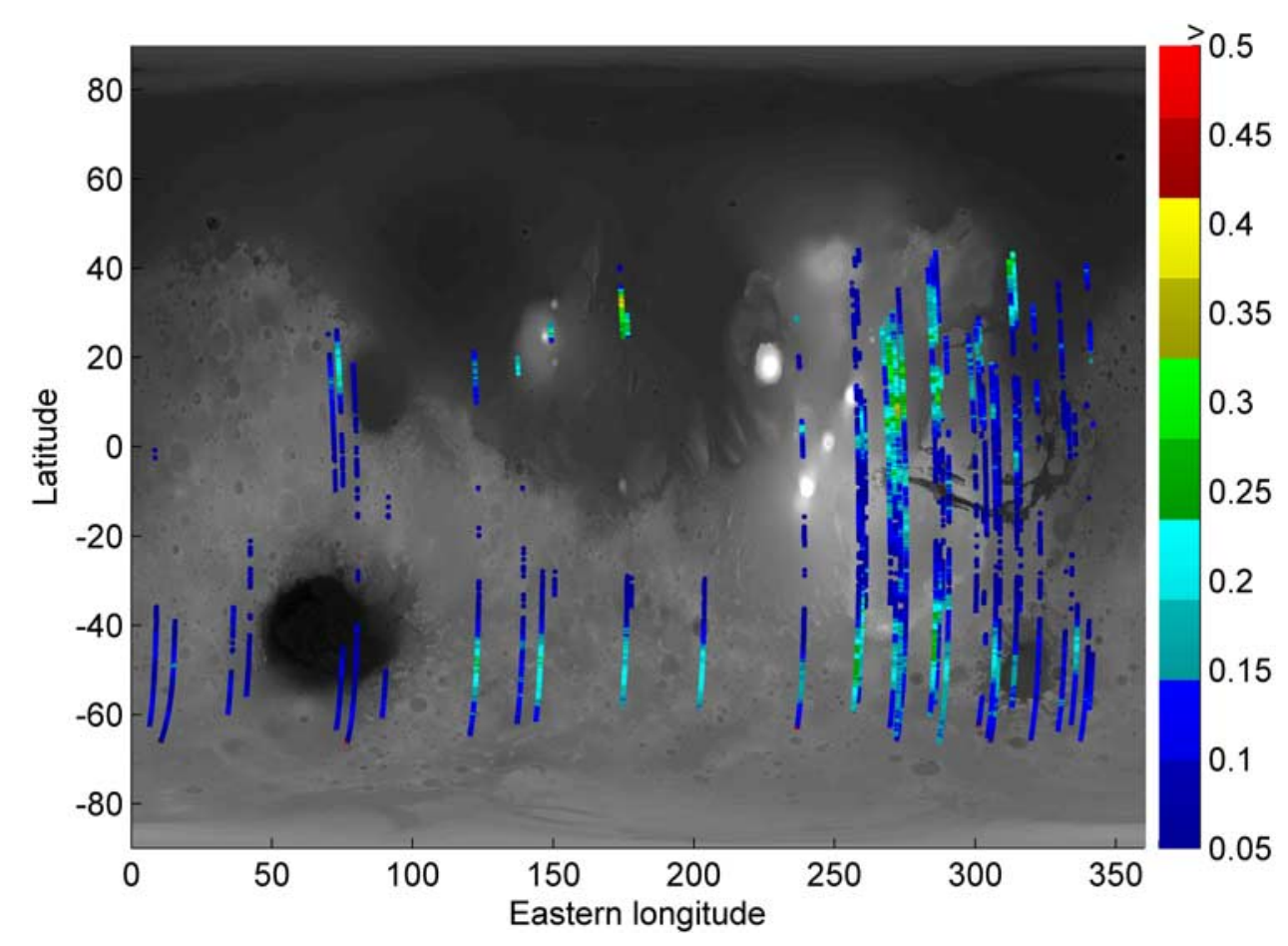

Figure 30. Cloudiness for $L_{s}=20-40^{\circ}$.

pronounced. After $L_{s}=50^{\circ}$ the orbital coverage was restricted (Figure 1). The orbits which penetrated south from $30^{\circ} \mathrm{S}$ occurred in the twilight zone. This did not allow us to register the edge of the south polar hood.

[71] The orbits attributed to $L_{s}=60-77^{\circ}$ were mainly acquired in morning or evening hours. Distinct hazes with optical thickness $\sim 0.1$ were observed above Lunae Planum and Amazonis Planitia at about 7 a.m. local time. Evening orbits showed an increase of cloudiness in the Tharsis region.

\subsubsection{Northern Summer}

[72] The aphelion cloud belt was the main cloud system observed during the northern summer. In the beginning of summer $\left(L_{s}=93^{\circ}\right)$ we found the ACB well developed (Figure 32 ). The belt was centered on $15^{\circ} \mathrm{N}$. It was very prominent in the Tharsis region, above the Lunae Planum, and Chryse Planitia. The other centers of intense cloudiness were Elysium Mons and Syrtis Major. Clouds were also registered above Arabia Terra and Amazonis Planitia. In the south, a boundary of clouds was registered in Hellas basin. After $L_{s}=140^{\circ}$ the ACB practically disappeared. Fedorova et al. [2006] retrieved water vapor column concentration from SPICAM IR channel measurements. According to their results the increase of water vapor supply at $15^{\circ} \mathrm{N}$, the latitude where ACB is centered, occurred from $L_{s}=$ $120^{\circ}$ to at least $L_{s}=200^{\circ}$ (after this date there was a gap in the measurements). The rising and decay time of the ACB did not coincide with the period of maximal water vapor abundance. Pearl et al. [2001] also observed such a displacement between the ACB and water vapor supply. Basing themselves on TES temperature measurements they supposed that the ACB development is rather driven by a temperature decrease around aphelion than by an increase of water vapor supply.
[73] Wolff et al. [1999] used HST observations to investigate ACB variations. They retrieved values of cloud optical thickness above some particular areas. The HST optical thickness above Syrtis Major varied from 0.1 to 0.2 at the wavelength $410 \mathrm{~nm}$. SPICAM measurements showed $\tau_{c}=0.1-0.35$ in the same time period. Clouds above Amazonis Planitia were $\tau_{c} \sim 0.1$ according to HST measurements and $0.1-0.2$ according to SPICAM measurements. Above Chryse Planitia results of HST were $\tau_{c}=$ $0.1-0.3$ and the same values are obtained from SPICAM.

[74] Some orographic clouds appeared to be a part of the ACB. The cloud above Olympus Mons (orbit 1177) observed at $L_{s}=131^{\circ}$ was the most remarkable cloud in the whole observational period, with $\tau_{c}=1.13$. Benson et al. [2003] used MOC blue images $(400-450 \mathrm{~nm})$ to retrieve optical thickness of some orographic clouds. The maximal cloud optical thickness which they observed above Olympus Mons was $1.015\left(L_{s}=104^{\circ}\right)$. They observed a slightly lower value of $\tau_{c}=0.793$ at $L_{s}=136^{\circ}$. The results are in good agreement with SPICAM measurements taking into account that we compare measurements of different years. Orbit 980 showed a prominent cloud above Elysium Mons with $\tau_{c}=0.68$. In the beginning of the considered period at $L_{s}=93-95^{\circ}$ clouds above Alba Patera $\left(\tau_{c}=0.2\right)$ were registered.

[75] Although the general reason of the ACB formation is the ascending branch of Hadley circulation [Clancy et al., 1996], the local cloud distribution may be driven by relief. Many clouds that form the ACB, correlate with a sharp increase of the surface altitude, although they are not associated with a particular mountain (Figure 19). In this context we should also point out that one of the places where cloudiness is frequently observed, Syrtis Major, borders on the deep Isidis Planitia, that may also have some 


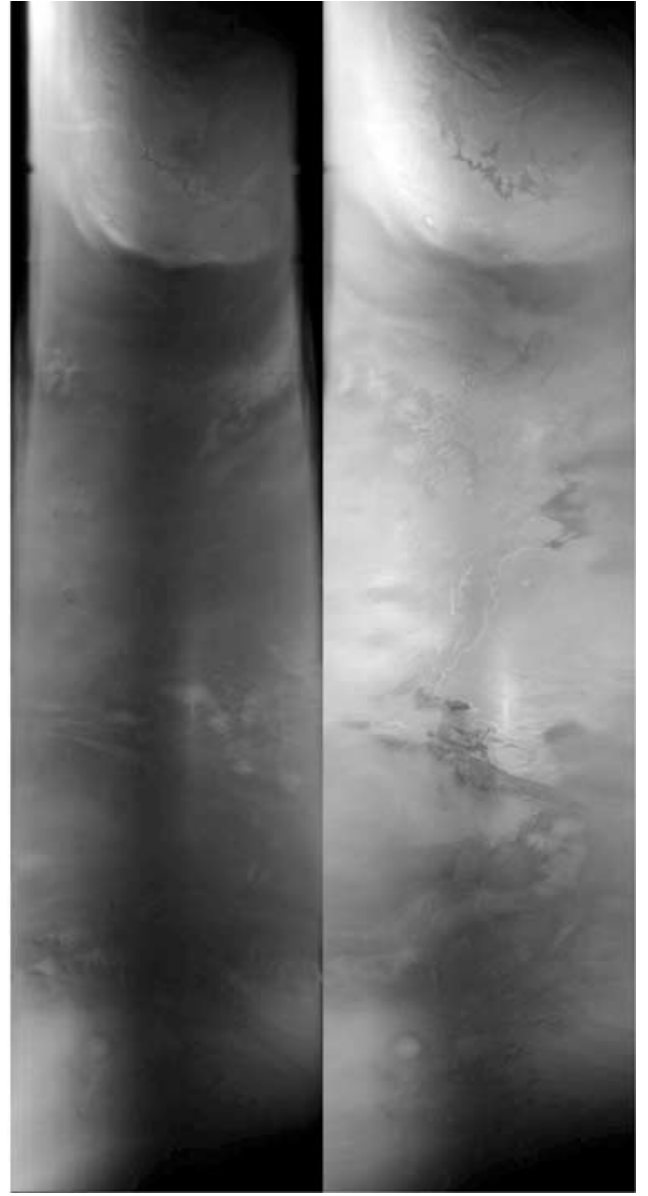

Figure 31. MOC (left) blue (R1700547) and (right) red (R1700546) images attributed to $L_{s}=30^{\circ}$ (7 May 2004), central longitude $284.2^{\circ} \mathrm{E}$, central latitude $15.30^{\circ} \mathrm{N}$. Slight haze which expands up to the north pole can be seen on the blue image.

influence on the cloud formation. The other important issue is a possible exchange of ice particles between clouds of Syrtis Major and the Hellas basin, because intensive clouds above both places were seen along the same orbits.

[76] The north polar summer clouds (NPSC) were observed in the period $L_{s}=110-130^{\circ}$ in the vicinity of the north pole (Figures 32 and 33). They were grouped mainly in the longitudinal region $230-330^{\circ} \mathrm{E}$. Tamppari et al. [2006] detected the NPSC in the same region using TES measurements. This longitudinal region corresponds to the most active part of the ACB above the Tharsis region (Figure 32). Figure 34 presents two examples of the NPSC, registered by the MOC. They were observed on the same days as the clouds detected in the orbits 1209 and 1096, respectively. Figure 34 shows that the NPSC detected by SPICAM are mainly parts of the large spiral cloud. This spiral cloud was described by Cantor et al. [2002] as a north annular cloud which appears every Martian year at approximately coincident dates.

\subsubsection{Northern Fall and Beginning of Winter}

[77] After summer break the north polar hood started to grow at $L_{s}=160^{\circ}$ (Figures 27 and 35). Figure 27 clearly shows a progress of the north polar hood southward during this period. After $L_{s}=180^{\circ}$ clouds again appeared above and in the vicinity of Alba Patera. The polar hood was not very dense in this early stage of its development and its optical thickness varied from 0.1 to 0.25 . Some weak orographic clouds above Olympus Mons, Arsia Mons and Ascreas Mons were also detected. A weak cloudiness was observed in the north of Valles Marineris and in the vicinity of the Hesperia Planum.

[78] In the second part of northern summer, after $L_{s}=$ $130^{\circ}$ some of orbits penetrated southward far enough to allow observing the edge of the south polar hood. The northeast latitude where the polar hood was observed was $40^{\circ} \mathrm{S}$ at $L_{s}=150^{\circ}$ (Figure 35). This is consistent with TES observations [Smith, 2004]. After $L_{s}=150^{\circ}$ the south polar hood retreated southward and was observed on the latitude $50^{\circ} \mathrm{S}$ at $L_{s}=190^{\circ}$. The optical thickness of the observed polar hood edge was $0.2-0.25$. The gap in measurements between $L_{s}=200^{\circ}$ and $L_{s}=240^{\circ}$ did not allow to observe a further development of the north polar hood and the retreat of the south polar hood during north fall.

[79] After $L_{s}=240^{\circ}$ the south polar hood disappeared. The orbit tracks shifted essentially southward and the north polar hood disappeared from the zone of observations. The only clouds observed between $L_{S}=240^{\circ}$ and $L_{S}=330^{\circ}$ were tiny clouds above Arsia Mons and Pavonis Mons. These clouds were usually series of narrow peaks at the northern or southern flanks of the volcanoes (Figure 10c).

\subsubsection{Water Content Estimate}

[80] The previous analysis considered only optical thicknesses of clouds. Following Benson et al. [2003] water content also could be estimated. Clancy et al. [2003] and Wolff and Clancy [2003] estimate the ice cloud particle effective radius as $1-2 \mu \mathrm{m}$ for clouds out of $\mathrm{ACB}$ and 3-4 $\mu \mathrm{m}$ for ACB. The Mie theory gives an estimate of the water ice extinction cross section $s_{\text {ext }}^{1}=2.63 \cdot 10^{-7} \mathrm{~cm}^{2}$ and $s_{\text {ext }}^{2}=1.09 \cdot 10^{-6} \mathrm{~cm}^{2}$ for a particle with effective radius $r_{1}=$ $2 \mu \mathrm{m}$ and $r_{2}=4 \mu \mathrm{m}$ respectively at wavelength $300 \mathrm{~nm}$. The optical properties of water ice were taken from Warren [1984]. For the thin clouds, the optical thickness can be estimated as $\tau_{c}=N s_{\text {ext }}$, where $N$ is the column number density of ice particles. The mass of water ice in a vertical column per unit area is expressed as $m=(4 / 3) \pi r^{3}\left(\tau_{c} / s_{\text {ext }}\right) \rho$, where $\rho=0.917 \mathrm{gcm}^{-3}$ is the water ice density. Finally we obtain coefficients $k_{1}=1.17\left(r_{1}=2 \mu \mathrm{m}\right)$ and $k_{2}=2.25\left(r_{2}=\right.$ $4 \mu \mathrm{m}$ ) for the conversion of the optical thickness to the water content which will be expressed in $\mathrm{gm}^{-2}$ or in $\operatorname{pr} \mu \mathrm{m}$. We use coefficient $k_{2}$ for well-developed ACB clouds and $k_{1}$ for other clouds. The water content in the ACB varied from 0.12 to $0.35 \mathrm{pr} \mu \mathrm{m}$ (that corresponds to optical thicknesses $0.1-0.3$ ) on the early stages of the belt formation at $L_{s}=20-40^{\circ}$ and increased up to $1.35-1.8 \mathrm{pr} \mu \mathrm{m}$ (optical thicknesses $0.6-0.8$ and $k_{2}$ were used) at $L_{s}=90-140^{\circ}$, when the belt had been well developed. The maximal value of $\tau_{c}=1.13$ registered above Olympus Mons at the orbit 1177 gave the water content estimate of $1.32 \mathrm{pr} \mu \mathrm{m}$ for $k_{1}$ and $2.54 \mathrm{pr} \mu \mathrm{m}$ for $k_{2}$. The optical thickness characteristic for the polar hoods was 0.35 corresponding to a water content of $0.40 \operatorname{pr} \mu \mathrm{m}$.

[81] Below we compare the water content estimates with the water vapor column abundance obtained by Fedorova et 


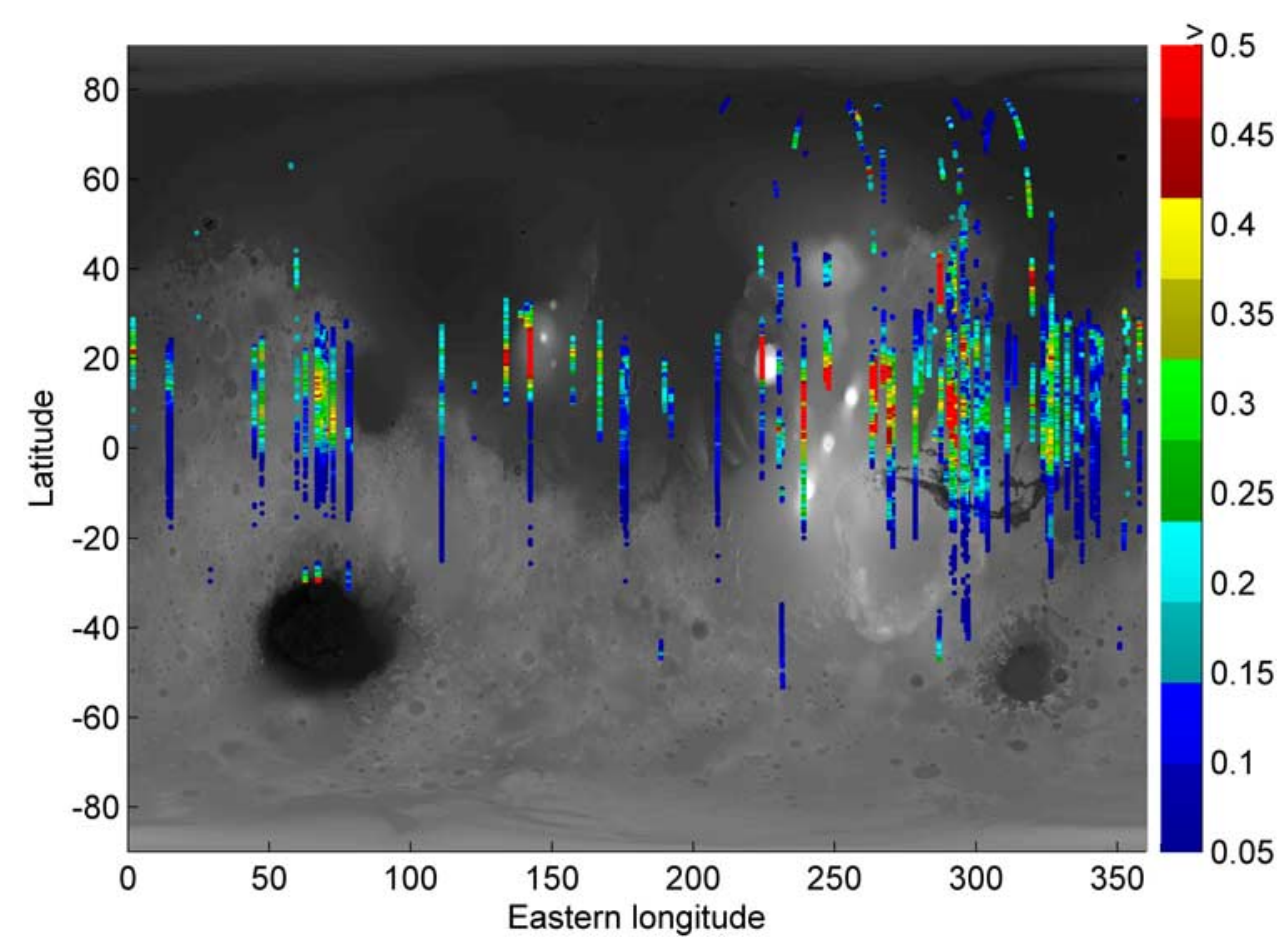

Figure 32. Equatorial cloud belt. $\mathrm{L}_{\mathrm{s}}=90-140^{\circ}$.

al. [2006] from SPICAM IR measurements. It is worth to mention that the results obtained by Fedorova et al. [2006] are systematically lower by factor $1.5-1.8$ than other instrument results, as the authors point it out.
[82] According to Fedorova et al. [2006], the water vapor column abundance in the north tropics during the presence of the ACB was 5-10 $\mathrm{pr} \mu \mathrm{m}$. The water content estimate for the typical ACB cloud optical depth $\tau_{c}=0.5$ is $1.13 \mathrm{pr} \mu \mathrm{m}$.
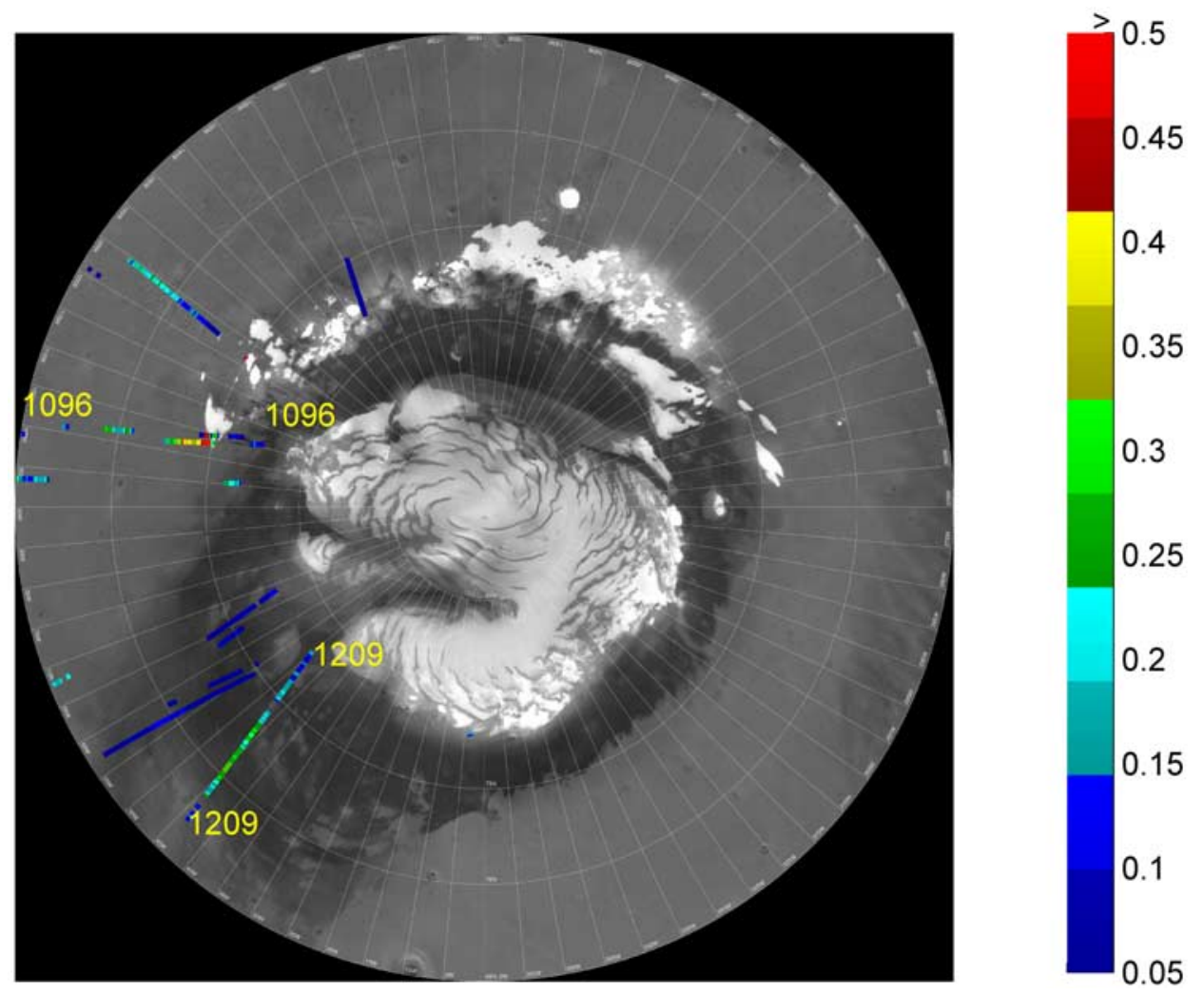

Figure 33. North summer clouds. $\mathrm{L}_{\mathrm{s}}=90-140^{\circ}$. 


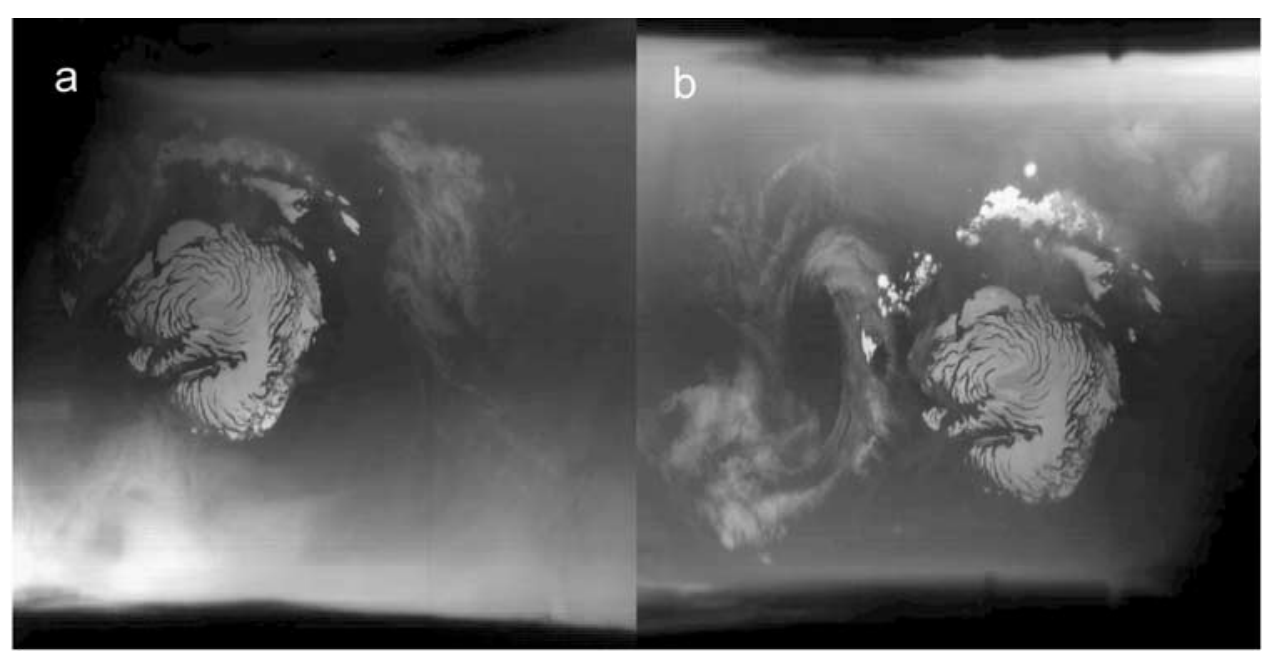

Figure 34. An example of a north polar cloud. (a) Blue MOC image S0101125, $L_{s}=135.5^{\circ}$ (27 December 2004). This cloud was detected by SPICAM at orbit 1209. (b) Blue MOC image R2301176, $L_{s}=120.02^{\circ}$ (25 November 2004). This cloud was detected by SPICAM at orbit 1096.

This means that only $10-20 \%$ of the total water content is stored within the clouds in this period. Both polar hoods are formed in conditions of lower water vapor content, about $1 \mathrm{pr} \mu \mathrm{m}$, and the polar hood clouds accumulate up to $30 \%$ of the total water content. The decay of the ACB was observed already after $L_{S}=140^{\circ}$, while the concentration of water vapor at the latitude of the $\mathrm{ACB}, 15^{\circ} \mathrm{N}$, continued to increase and enhanced till $L_{s}=200^{\circ}$ [Fedorova et al.,2006]. The observed time shift between the maximal development of the ACB and maximal water vapor abundance is in agreement with TES results [Smith, 2004] and supports their conclusion that the ACB formation is rather connected with low air temperatures observed in the same period than the water vapor abundance.

\section{Conclusions}

[83] In this study we investigated the Martian water ice cloud distribution using the SPICAM UV spectrometer on board Mars Express. The considered data set covers nearly one Martian year (MY 27) from January 2004 to January 2006. The ability of SPICAM to detect ice clouds arises from the fact that Martian bare ground has a very low albedo in the UV $(0.004-0.018)$. Ice clouds manifest themselves as a prominent increase of the signal and they

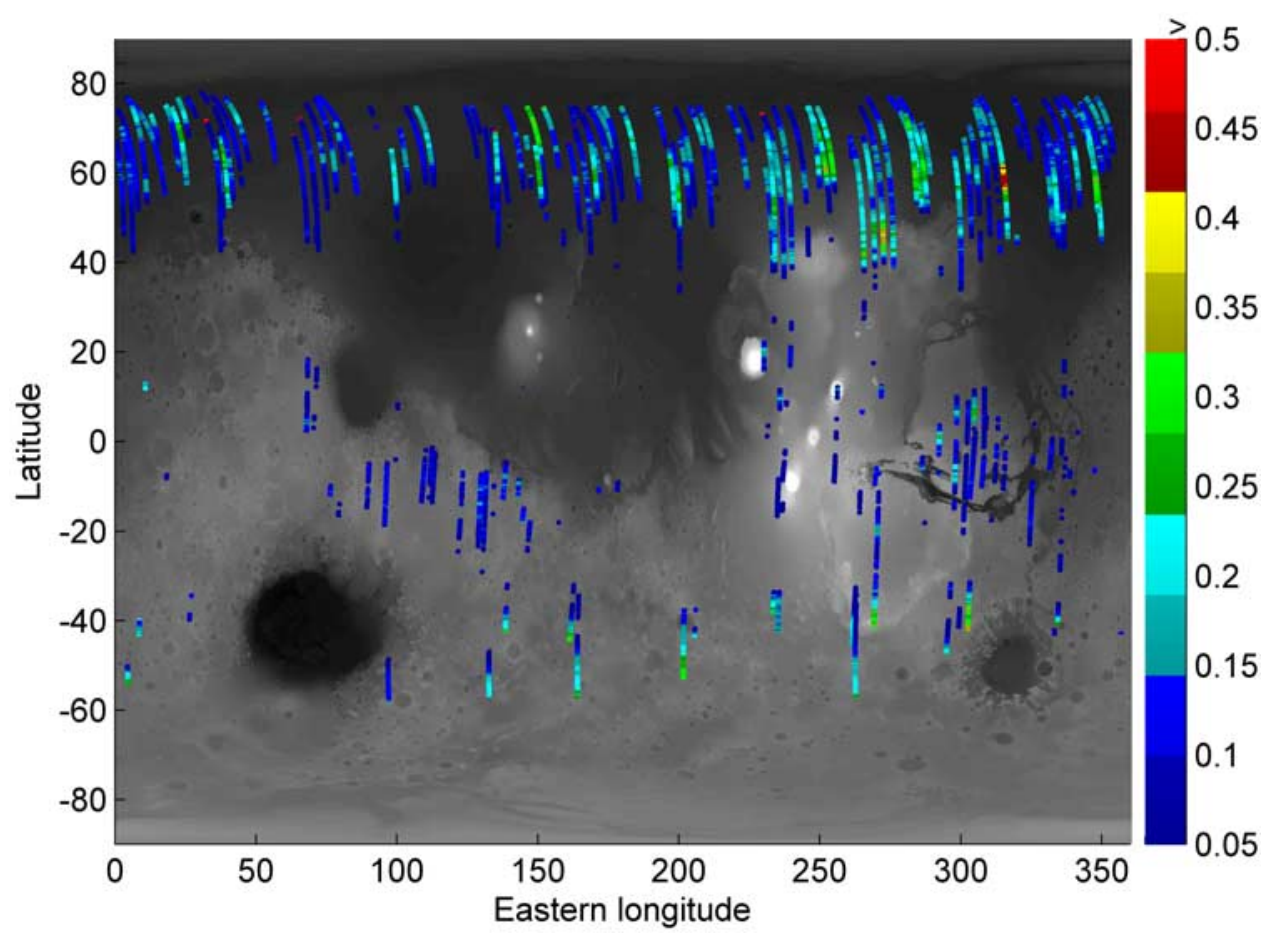

Figure 35. Cloudiness for $\mathrm{L}_{\mathrm{s}}=150-200^{\circ}$. 
could be separated from the bright seasonal polar ices using MOC and TES observations of the ice boundaries.

[84] The wavelength range of the SPICAM UV channel (118-308 nm) includes the Hartley ozone absorption band centered at $250 \mathrm{~nm}$. Therefore, in order to exclude ozone interference from the ice cloud retrieval procedure, only a narrow wavelength range centered at $300 \mathrm{~nm}$ was considered.

[85] The optical properties of Martian dust are not well known in the UV. To include dust correctly in the forward model a dust storm observed at October 2005 was investigated. The analysis showed that the dust presence effect strongly depends on the wavelength. The dust clouds are bright in the visible [e.g., Cantor et al., 2001] and become dark in the UV showing strong extinction. The effect of dust appeared to be minimal around the wavelength $300 \mathrm{~nm}$. This allowed to exclude dust from the forward model for the ice cloud optical depth retrieval.

[86] The modeling of the conditions at which the radiance factor becomes minimally sensitive to the increase of the dust optical thickness produced to estimate of the dust asymmetry factor $g_{d}=0.8$ at $300 \mathrm{~nm}$ if we adopt the dust single scattering albedo $\sigma_{d}=0.6$ from Wolff et al. [1999]. Of course, the final conclusion about the dust optical properties in the UV needs more detailed analysis which is out of the scope of this paper.

[87] The important factor to be considered in the retrieval of the ice cloud optical thickness is the surface albedo. To build the map of the surface albedo at $300 \mathrm{~nm}$ an "apparent" albedo was first retrieved without making difference between clouds and bright surface features. The nonLambertian properties of the surface albedo at low values of phase angles were corrected using the Hapke theory [Hapke, 1986]. The "apparent" albedo values were classified by excluding the higher values attributed to clouds and ices. The values which were kept were then statistically processed for different territories. The resultant albedo map showed significant anticorrelation with the visual albedo of the Martian surface. This is consistent with the distribution of Martian minerals obtained by the OMEGA instrument [Bibring et al., 2006]. The territories which look bright in the visible range are abundant in ferric oxides [Bibring et al., 2006] that absorb strongly in the UV causing the anticorrelation effect.

[88] The main results of this work are the maps of the ice cloud optical thickness distribution. The ice cloud optical thicknesses were retrieved using the precomputed albedo map. In most cases, the clouds are well contrasted with respect to the background.

[89] The aphelion cloud belt (ACB) is the most prominent cloud feature. The formation of the belt starts in early spring at $L_{s}=20^{\circ}$ by a cloudiness increase eastward from the Tharsis region and Lunae Planum. This period is characterized by low air temperatures [Smith, 2004]. At $L_{s}=30^{\circ}$ thin clouds form a "bridge" between the clouds located in the north tropics and the south polar hood in the longitudinal sector $250-340^{\circ} \mathrm{E}$.

[90] The ACB is significantly enhanced in the period $L_{s}=$ $93-140^{\circ}$ and after $L_{s}=140^{\circ}$ quickly decays. In the early stage of formation, the characteristic values of the cloud optical thickness are between 0.1 and 0.3 . When the belt is already well developed, the maximal values of the optical thickness vary between $0.6-0.8$ and sometimes reach 1.13 . The period of maximal development of the ACB does not coincide with the period of maximal concentration of water vapor [Fedorova et al., 2006; Smith, 2004] and is rather connected with low air temperatures observed in the same period [Smith, 2004].

[91] The northern polar summer clouds were observed in the same period as the ACB. Many of them are parts of the spiral clouds observed by MOC [Cantor et al., 2002]. The clouds mostly appeared in the longitudinal sector 250$270^{\circ} \mathrm{E}$ between the north pole and the prominent Tharsis highland.

[92] Both polar hoods were observed at the stages of their development and decay. The observed cloud optical thicknesses are lower that in the ACB and in general do not exceed 0.35 . The north polar hood starts to develop at $L_{s}=$ $160^{\circ}$ and the south at $L_{s}=330^{\circ}$. The edge of the south polar hood was observed also during the northern summer between $L_{s}=130^{\circ}$ and $L_{s}=200^{\circ}$.

[93] By using the retrieved cloud optical depth, the water column abundances were estimated. The water content varies from 0.1 to $1.8 \mathrm{pr} \mu \mathrm{m}$. According to Fedorova et al. [2006] the water vapor column abundance in the north tropics during the presence of the ACB is 5-10 $\mathrm{pr} \mu \mathrm{m}$. This means that only $10-20 \%$ of the total water content is stored into clouds in this period. Both polar hoods are formed in conditions of lower water vapor content, about $1 \mathrm{pr} \mu \mathrm{m}$, and the polar hood clouds accumulate up to $30 \%$ of the total water content.

[94] Acknowledgments. This work was supported by the Belgian Scientific Policy office under grant MO/035-017. We thank our reviewers, M. Wolff and M. Smith, for thorough and constructive comments.

\section{References}

Bell, J. F., III (1996), Iron, sulfate, carbonate, and hydrated minerals on Mars, in Mineral Spectroscopy: A Tribute to Roger G. Burns, edited by M. D. Dyar, C. McCammon, and M. W. Schaefer, Spec. Publ. Geochem. Soc., 5, 359-380.

Benson, J. L., and P. B. James (2005), Yearly comparisons of the martian polar caps: 1999-2003 Mars Orbiter Camera observations, Icarus, $174(2), 513-523$

Benson, J. L., B. P. Bonev, P. B. James, K. J. Shan, B. A. Cantor, and M. A Caplinger (2003), The seasonal behavior of water ice clouds in the Tharsis and Valles Marineris regions of Mars: Mars Orbiter Camera observations, Icarus, 165, 34-52.

Bertaux, J. L., et al. (2000), The study of the martian atmosphere from top to bottom with SPICAM light on Mars Express, Planet. Space Sci., 48, $1303-1320$

Bertaux, J.-L., et al. (2006), SPICAM on Mars Express: Observing modes and overview of UV spectrometer data and scientific results, J. Geophys. Res., 111, E10S90, doi:10.1029/2006JE002690.

Bibring, J. P., et al. (2006), Global mineralogical and aqueous Mars history derived from OMEGA/Mars Express data, Science, 312, 400-404.

Cantor, B. A., P. B. James, M. Caplinger, and M. J. Wolff (2001), Martian dust storms: 1999 Mars orbiter camera observations, J. Geophys. Res., 106(E10), 23,653-23,687.

Cantor, B., M. Malin, and K. S. Edgett (2002), Multiyear Mars Orbiter Camera (MOC) observations of repeated Martian weather phenomena during the northern summer season, J. Geophys. Res., 107(E3), 5014, doi:10.1029/2001JE001588.

Christensen, P. R., et al. (2001), Mars Global Surveyor Thermal Emission Spectrometer experiment: Investigation description and surface science results, J. Geophys. Res., 106(E10), 23,823-23,871.

Clancy, R. T., and S. W. Lee (1991), A new look at dust and clouds in the Mars atmosphere: Analysis of emission-phase-function sequences from global Viking IRTM observations, Icarus, 93, 135-158.

Clancy, R. T., A. W. Grossman, M. J. Wolff, P. B. James, D. J. Rudy, Y. N. Billawala, B. J. Sandor, S. W. Lee, and D. O. Muhleman (1996), Water 
vapor saturation at low latitudes around aphelion: A key to Mars climate?, Icarus, 122, 36-62.

Clancy, R. T., M. J. Wolff, and P. B. James (1999), Minimal aerosol loading and global increases in atmospheric ozone during the 1996-1997 martian northern spring season, Icarus, 138, 49-63.

Clancy, R. T., B. J. Sandor, M. J. Wolff, P. R. Christensen, M. D. Smith, J. C. Pearl, B. J. Conrath, and R. J. Wilson (2000), An intercomparison of ground-based millimeter, MGS TES, and Viking atmospheric temperature measurements: Seasonal and interannual variability of temperatures and dust loading in the global Mars atmosphere, J. Geophys. Res., 105, 9553-9572.

Clancy, R. T., M. J. Wolff, and P. R. Christensen (2003), Mars aerosol studies with the MGS TES emission phase function observations: Optical depths, particle sizes, and ice cloud types versus latitude and solar longitude, J. Geophys. Res., 108(E9), 5098, doi:10.1029/2003JE002058.

Conrath, B. J. (1975), Thermal structure of the Martian atmosphere during the dissipation of the dust storm of 1971, Icarus, 24, 36-46.

Evans, K. F. (1998), The spherical harmonics discrete ordinate method for three-dimensional atmospheric radiative transfer, J. Atmos. Sci., 55, 429446.

Fedorova, A., O. Korablev, J.-L. Bertaux, A. Rodin, A. Kiselev, and S. Perrier (2006), Mars water vapor abundance from SPICAM IR spectrometer: Seasonal and geographic distributions, J. Geophys. Res., 111, E09S08, doi:10.1029/2006JE002695.

Forget, F., F. Hourdin, R. Fournier, C. Hourdin, O. Talagrand, M. Collins, S. R. Lewis, P. L. Read, and J.-P. Huot (1999), Improved general circulation models of the Martian atmosphere from the surface to above $80 \mathrm{~km}$, J. Geophys. Res., 104(E10), 24,155-24,176.

Hapke, B. (1986), Bidirectional reflectance spectroscopy. 4. The extinction coefficient and the opposition effect, Icarus, 67, 264-280.

James, P. B., R. T. Clancy, S. W. Lee, L. J. Martin, R. B. Singer, E. Smith, R. A. Kahn, and R. W. Zurek (1994), Monitoring Mars with the Hubble Space Telescope: 1990-1991 observations, Icarus, 109, 79-101.

Jaquin, F., P. Gierasch, and R. Kahn (1986), The vertical structure of limb hazes in the Martian atmosphere, Icarus, 68, 442-461.

Key, J. R., P. Yang, B. A. Baum, and S. L. Nasiri (2002), Parameterization of shortwave ice cloud optical properties for various particle habits, J. Geophys. Res., 107(D13), 4181, doi:10.1029/2001JD000742.

Liou, K. N. (2002), An Introduction to Atmospheric Radiation, 2nd ed., Int. Geophys. Ser, vol. 84, 583 pp., Academic Press, San Diego, Calif.

McKim, R. (2006), Mars in 2005: First interim report, J.Br. Astron. Assoc., $116(1), 6$.

Montmessin, F., F. Forget, P. Rannou, M. Cabane, and R. M. Haberle (2004), Origin and role of water ice clouds in the Martian water cycle as inferred from a general circulation model, J. Geophys. Res., 109, E10004, doi:10.1029/2004JE002284

Pang, K., and J. M. Ajello (1977), Complex refractive index of Martian dust: Wavelength dependence and composition, Icarus, 30, 63-74.

Pang, K., J. M. Ajello, C. W. Hord, and W. G. Egan (1976), Complex refractive index of Martian dust: Mariner 9 ultraviolet observations, Icarus, 27, 55-67.

Pearl, J. C., M. D. Smith, B. J. Conrath, J. L. Bandeld, and P. R. Christensen (2001), Observations of Martian ice clouds by the Mars Global Surveyor Thermal Emission Spectrometer: The first Martian year, J. Geophys. Res., 106, 12,325-12,338

Perrier, S., J. L. Bertaux, F. Lefevre, S. Lebonnois, O. Korablev, A. Fedorova, and F. Montmessin (2006), Global distribution of total ozone on Mars from
SPICAM/MEX UV measurements, J. Geophys. Res., 111, E09S06, doi:10.1029/2006JE002681.

Petrova, E. H., U. Keller, W. J. Markiewicz, N. Thomas, and M. W. Wuttke (1996), Ice hazes and clouds in the Martian atmosphere as derived from the Phobos/KRFM data, Planet. Space Sci., 44, 1163-1176.

Richardson, M. I., and R. J. Wilson (2002), Investigation of the nature and stability of the Martian seasonal water cycle with a general circulation model, J. Geophys. Res., 107(E5), 5031, doi:10.1029/2001JE001536.

Richardson, M. I., R. J. Wilson, and A. V. Rodin (2002), Water ice clouds in the Martian atmosphere: General circulation model experiments with a simple cloud scheme, J. Geophys. Res., 107(E9), 5064, doi:10.1029/ 2001JE001804.

Rodin, A. V., O. I. Korablev, and V. I. Moroz (1997), Vertical distribution of water in the near-equatorial troposphere of Mars: Water vapor and clouds, Icarus, 125, 212-229.

Smith, D., G. Neumann, R. E. Arvidson, E. A. Guinness, and S. Slavney (2003), Mars Global Surveyor Laser Altimeter Mission Experiment Gridded Data Record, MGS-M-MOLA-5-MEGDR-L3-V1.0, http:// pds.jpl.nasa.gov/, NASA Planet. Data Syst., Washington, D. C.

Smith, M. D. (2002), The annual cycle of water vapor on Mars as observed by the Thermal Emission Spectrometer, J. Geophys. Res., 107(E11), 5115, doi:10.1029/2001JE001522.

Smith, M. D. (2004), Annual variability in TES atmospheric observations of Mars during 1999-2003, Icarus, 167, 148-165.

Smith, M. D., J. L. Bandfield, P. R. Christensen, and M. I. Richardson (2003), Thermal Emission Imaging System (THEMIS) infrared observations of atmospheric dust and water ice cloud optical depth, J. Geophys. Res., 108(E11), 5115, doi:10.1029/2003JE002115.

Tamppari, L. K., R. W. Zurek, and D. A. Paige (2000), Viking era water-ice clouds, J. Geophys. Res., 105, 4087-4107.

Tamppari, L. K., M. D. Smith, D. S. Bass, and A. S. Hale (2006), Water vapor, water-ice clouds, and dust in the North Polar Region, paper presented at Second Workshop on Mars Atmosphere Modelling and Observations, Cent. Natl. d'Etud. Spatiales, Granada, Spain.

Thorpe, T. E. (1982), Martian surface properties indicated by the opposition effect, Icarus, 49, 398-415.

Titus, T. N. (2005), Mars polar cap edges tracked over 3 full mars years, Lunar Planet. Sci., XXXVI, abstract 1993.

Wang, H., and A. P. Ingersoll (2002), Martian clouds observed by Mars Global Surveyor Mars Orbiter Camera, J. Geophys. Res., 107(E10), 5078, doi:10.1029/2001JE001815.

Warren, S. G. (1984), Optical properties of ice from the ultraviolet to the microwave, Appl. Opt., 23, 1206-1225.

Wolff, M. J., and R. T. Clancy (2003), Constraints on the size of Martian aerosols from Thermal Emission Spectrometer observations, J. Geophys. Res., 108(E9), 5097, doi:10.1029/2003JE002057.

Wolff, M. J., J. F. Bell, P. B. James, R. T. Clancy, and S. W. Lee (1999), Hubble Space Telescope observations of the Martian aphelion cloud belt prior to the Pathfinder, J. Geophys. Res., 104(E4), 9027-9041.

J.-L. Bertaux, E. Dimarellis, F. Montmessin, and S. Perrier, Service d'Aeronomie du CNRS/IPSL, F-91371 Verrières-le-Buisson, France.

C. Bingen, J. Dodion, D. Fussen, N. Mateshvili, and F. Vanhellemont, Belgian Institute for Space Aeronomy, B-1180 Brussels, Belgium. (ninam@oma.be) 